Journal for ImmunoTherapy of Cancer

\title{
CD4 T cell-intrinsic STING signaling controls the differentiation and effector functions of $T_{H} 1$ and $T_{H} 9$ cells
}

Isis Benoit-Lizon (D) , ${ }^{1,2}$ Elise Jacquin (1) ,1,2,3 Thaiz Rivera Vargas, ${ }^{1,2}$

Corentin Richard, ${ }^{1,2}$ Aurélie Roussey, ${ }^{1,2}$ Ludivine Dal Zuffo, ${ }^{1,2}$ Tiffany Martin,, ${ }^{1,2}$ Andréa Melis, ${ }^{1,2}$ Daria Vinokurova, ${ }^{1,2}$ Sayyed Hamed Shahoei, ${ }^{1,2,4}$ Alvaro Baeza Garcia (1) ,1,2 Cassandre Pignol, ${ }^{1,2}$ Stéphane Giorgiutti, ${ }^{5}$ Raphaël Carapito, ${ }^{6}$ Romain Boidot, ${ }^{1,2,7}$ Frédérique Végran, ${ }^{1,2,7}$ Richard A Flavell, ${ }^{8}$ Bernhard Ryffel, ${ }^{9,10}$ Eric R Nelson, ${ }^{4,11,12}$ Pauline Soulas-Sprauel, ${ }^{13}$ Toby Lawrence, ${ }^{14}$ Lionel Apetoh (1) 1,2,15,16

To cite: Benoit-Lizon I, Jacquin E, Rivera Vargas T, et al. CD4 T cell-intrinsic STING signaling controls the differentiation and effector functions of $T_{H} 1$ and $T_{H} 9$ cells. Journal for ImmunoTherapy of Cancer 2022;10:e003459. doi:10.1136/jitc-2021-003459

- Additional supplemental material is published online only. To view, please visit the journal online (http://dx.doi.org/10. 1136/jitc-2021-003459).

IB-L, EJ and TRV are joint first authors.

Accepted 02 December 2021

Check for updates

(C) Author(s) (or their employer(s)) 2022. Re-use permitted under CC BY. Published by BMJ.

For numbered affiliations see end of article.

Correspondence to

Pr Lionel Apetoh;

lionel.apetoh@inserm.fr

\section{ABSTRACT}

Background While stimulator of interferon genes (STING) activation in innate immune cells of the tumor microenvironment can result in CD8 T cell-dependent antitumor immunity, whether STING signaling affects CD4 T-cell responses remains elusive.

Methods Here, we tested whether STING activation modulated the effector functions of CD4 T cells in vivo by analyzing tumor-infiltrating CD4 T cells and evaluating the contribution of the CD4 T cell-derived cytokines in the antitumor activity of the STING ligand $2^{\prime} 3^{\prime}$-cyclic guanosine monophosphate-adenosine monophosphate (cGAMP) in two mouse tumor models. We performed ex vivo experiments to assess the impact of STING activation on CD4 T-cell differentiation and investigate the underlying molecular mechanisms. Finally, we tested whether STING activation enhances $\mathrm{T}_{\mathrm{H}} 9$ cell antitumor activity against mouse melanoma upon adoptive transfer.

Results We found that activation of STING signaling cell-intrinsically enhances the differentiation and antitumor functions of $T_{H} 1$ and $T_{H} 9$ cells by increasing their respective production of interferon gamma (IFN- $\gamma$ ) and interleukin-9. IRF3 and type I interferon receptors (IFNARs) are required for the STING-driven enhancement of $T_{H} 1$ cell differentiation. However, STING activation favors $\mathrm{T}_{H} 9$ cell differentiation independently of the IFNARs/IRF3 pathway but through mammalian target of rapamycin (mTOR) signaling, underscoring that STING activation differentially affects the fate of distinct CD4 T-cell subsets. The therapeutic effect of STING activation relies on $T_{H} 1$ and $T_{H}$ 9-derived cytokines, and STING activation enhances the antitumor activity of $\mathrm{T}_{\mathrm{H}} 9$ cells upon adoptive transfer. Conclusion Our results reveal the STING signaling pathway as a therapeutic target to boost CD4 T-cell effector functions and antitumor immunity.

\section{INTRODUCTION}

CD4 effector $\mathrm{T}$ cells can make decisive contributions to antitumor immunity. This is underscored not only by preclinical studies indicating that activation of CD4 $\mathrm{T}$ cells in the tumor microenvironment is required for productive antitumor immune responses and tumor clearance, ${ }^{1}$ but also by clinical investigations showing that the adoptive transfer of antigen-specific CD4 T cells into melanoma patients can lead to remission. ${ }^{2}$ Following the characterization of $\mathrm{T}_{\mathrm{H}} 1$ cells as interferon gamma (IFN- $\gamma$ )-producing cells, which are differentiated from naive CD4 $\mathrm{T}$ cells in the presence of interleukin (IL)-12, ${ }^{3}$ the antitumor functions of these cells were exemplified in mouse and human cancers. More recently, the IL-9-secreting $\mathrm{T}_{\mathrm{H}} 9$ cells were characterized as another effector T-cell subset with potent antitumor properties. We and others have shown that these cells exert IL-9-mediated antitumor functions upon adoptive transfer in melanoma-bearing mice, identifying them as potential effector $\mathrm{T}$ cells for adoptive cell therapy of cancer. ${ }^{4-6}$ $\mathrm{T}_{\mathrm{H}} 9$ cells were originally shown to differentiate from naive $\mathrm{CD} 4 \mathrm{~T}$ cells stimulated with transforming growth factor beta (TGF- $\beta$ ) and IL- $4 .{ }^{7}$ Proinflammatory factors including IL-1 $\beta$ and tumor necrosis factor alpha (TNF- $\alpha$ ) enhance $\mathrm{T}_{\mathrm{H}} 9$ differentiation. ${ }^{5} 8-10$ A growing number of studies also indicate that $\mathrm{T}_{\mathrm{H}} 9$ cells are essential for the efficacy of cancer immunotherapy treatments such as dendritic cell (DC) vaccination and antiglucocorticoid-induced tumor necrosis factor receptor (GITR) therapy. ${ }^{11} 12$

The differentiation of naive $\mathrm{CD} 4 \mathrm{~T}$ cells requires T-cell receptor (TCR)-driven, costimulatory, and cytokine-derived signals. These signals can be provided by activated innate immune myeloid cells such as DCs, which drive T-cell activation and polarization and thus dictate the magnitude and 
quality of adaptive immune responses. ${ }^{13}$ The detection of danger signals from invading viruses or bacteria or from damaged autologous cells drives DC maturation and cytokine secretion. ${ }^{14}$ The triggering of cytosolic receptors in innate immune cells leads to activation of the adaptor protein stimulator of interferon genes (STING) ${ }^{15}$ which results in the induction of a specific transcriptional program that culminates in the expression of type I interferons (IFNs) and proinflammatory cytokines. ${ }^{16}$ The administration of the STING ligand cyclic guanosine monophosphate-adenosine monophosphate (hereafter referred to as cGAMP) into solid tumors results in the induction of anticancer immune responses that promote tumor control. ${ }^{17-19}$ Likewise, the systemic delivery of a small-molecule STING agonist induces potent CD8 T celldependent anticancer immunity in vivo in mice. ${ }^{20}$ The clinical potential of STING targeting in solid tumors is currently being evaluated. ${ }^{21}$

While the functions of STING have been extensively studied in myeloid cells, we and others documented that STING is also expressed in CD4 T cells. ${ }^{22-24}$ Potent STING activation using synthetic ligands has been proposed to trigger T-cell death through type I-dependent and type I-independent pathways. ${ }^{24-27}$ However, the consequences of CD4 T cell-intrinsic STING activation on their differentiation and effector responses remain unclear. Here we report that the activation of STING in differentiating $\mathrm{T}_{H} 1$ and $\mathrm{T}_{\mathrm{H}} 9$ cells enhances their respective secretion of IFN- $\gamma$ and IL-9, resulting in enhanced anticancer activity in vivo. Our results uncover STING as a molecular target to modulate CD4 T-cell differentiation and functions for therapeutic use.

\section{METHODS \\ Mice}

Wild type (WT) female C57BL/6 mice were purchased from Charles River laboratories (France). $\mathrm{Irf}^{-1-}\left(\mathrm{IRF}^{-/-}\right.$, RBRC00858 - RIKEN), Rela ${ }^{\mathrm{fl} / \mathrm{fl}}$ (p65, 024342, The Jackson Laboratory), Cd4-Cre (017336, The Jackson Laboratory), IL-9-Green Fluorescent Protein (GFP) ${ }^{28}$ Sting $^{-/-}$, OT-II (004194, The Jackson Laboratory), Trp1 (008684, The Jackson Laboratory), and Ifnar ${ }^{-1-}$ C57BL/6 mice were all bred at the Transgénèse et Archivage d'Animaux Modèles (TAAM, Orléans, France). IL-9-GFP as well as Sting $1^{-/-}$ and type I interferon receptor $(\text { IFNAR) })^{-/-}$mice were respectively provided by Professor Richard Flavell and Professor Bernhard Ryffel. STING V154M/WT ${ }^{29}$ mice and control littermates were kindly provided by Professor Soulas-Sprauel.

For in vivo experiments, 6-12 week-old female mice were matched by age and randomly assigned to specific treatment groups except for $\mathrm{Rag}^{-/-} \mathrm{STING}^{-/-}$experiments which include male mice equally distributed among the different treatment groups.

For in vitro experiments, 6-12 week-old mice were matched by age and sex. All transgenic mice used were on a C57BL/6 background and were age-matched with WT controls for experiments.

\section{Animal procedures}

Cell lines and tumor growth experiments

B16-F10, B16-OVA (B16-F10 cells engineered to express OVA) mouse melanoma, MC38 and MC38-OVA (MC38 cells engineered to express OVA) mouse colon adenocarcinoma cell lines were cultured at $37^{\circ} \mathrm{C}$ under $5 \%$ $\mathrm{CO}_{2}$ in the following culture media. B16-F10 were maintained in Dulbecco's Modified Eagle Medium (DMEM) high glucose (Dutscher) supplemented with $10 \%$ (vol/ vol) heat-inactivated fetal bovine serum (FBS, Dutscher) and $100 \mathrm{U} / \mathrm{mL}$ penicillin, $0.1 \mathrm{mg} / \mathrm{mL}$ streptomycin, $0.25 \mu \mathrm{g} / \mathrm{mL}$ amphotericin B (1\%Penicillin Streptomycin Amphotericin B, PSA, Pan Biotech). B16-OVA, MC38 and MC38-OVA were respectively maintained in Roswell Park Memorial Institute (RPMI) $1640 \mathrm{w} / \mathrm{L}$-glutamine or DMEM high glucose containing $10 \mathrm{mM}$ Hepes (Gibco) supplemented with $10 \%$ (vol/vol) heat-inactivated FBS, 1\% PSA, $1 \mathrm{mM}$ sodium pyruvate (Gibco), $2 \mathrm{mM}$ L-glutamine (Gibco) and Minimum Essential MediumNon-Essential Amino Acids (MEM NEAA) (0.1 mM each AA, Gibco). B16-F10 and MC38 cells were respectively obtained from American Type Culture Collection (ATCC) and Kerafast. B16-OVA and MC38-OVA cells were respectively kindly provided by Professor Yong $\mathrm{Lu}$ (Department of Microbiology and Immunology, Wake Forest School of Medicine, Winston-Salem, North Carolina, USA) and Professor Ana Anderson (Harvard Medical School, Boston, USA).

Tumor cells $\left(1.5 \times 10^{5}\right.$ B16-F10 or B16-OVA cells per mouse, $5 \times 10^{5}$ MC38 or MC38-OVA cells per mouse) were resuspended in sterile phosphate buffered saline (PBS) and implanted subcutaneously. Each mouse received two intratumoral injections of PBS containing $100 \mu \mathrm{g}$ of cGAMP $\left(\mathrm{G}\left(2^{\prime}, 5^{\prime}\right) \mathrm{pA}\left(3^{\prime}, 5^{\prime}\right) \mathrm{p}\right.$, Invivogen) encapsulated in 2,5 $\mu \mathrm{L}$ of Lipofectamine 2000 (Invitrogen) or PBS with Lipofectamine 2000 alone (control) on days 5 and 10.

As for the $\mathrm{Rag}^{-/-} \mathrm{STING}^{-/-}$experiment, mice were injected intravenously with $5 \times 10^{6} \mathrm{WT}$ or $\mathrm{STING}^{-/-} \mathrm{CD} 4$ $\mathrm{T}$ cells and $2.5 \times 10^{6} \mathrm{STING}^{-/-} \mathrm{CD} 8 \mathrm{~T}$ cells, respectively, isolated with CD4 (L3T4) and CD8 (Ly-2) MicroBeads (Miltenyi Biotec) according to the manufacturer's instructions. MC38 tumor implantations were performed 5 weeks post- $T$ cell transfer and mice were treated as described above.

Tumor length and width were measured three times a week using calipers. Mice with tumor size exceeding 300 $\mathrm{mm}^{2}$ or with ulcerated tumors were euthanized in agreement with ethical guidelines.

Alternatively, for tumor-infiltrating lymphocyte (TIL) analysis, MC38 tumor-bearing mice were treated intratumorally when tumors reached $40-50 \mathrm{~mm}^{2}$ with cGAMP (50 $\mu \mathrm{g}$ per mouse) or PBS with Lipofectamine 2000 alone (control) and tumors were harvested 1 day after treatment. 
As for TIL analysis in MC38-OVA tumor-bearing mice, mice were injected intravenously with OT-II CD4 T cells $\left(6 \times 10^{6}\right) 4$ days before MC38-OVA subcutaneous injection.

\section{Cytokine neutralization}

Cytokine neutralization was achieved by intraperitoneal injections (200 $\mu \mathrm{g}$ per mouse) of anti-IFN- $\gamma$ (clone XMG1.2, BioXCell), anti-IL-9 (clone 9C1, BioXCell), antiIL-4 (clone 11B11, BioXCell) or anti-IL-17 (clone 17F3, BioXCell) antibodies 1 day before tumor cell implantation and then three times a week. Alternatively, IFN- $\gamma$ neutralization in MC38 tumor-bearing mice was achieved by intraperitoneal injections ( $200 \mu \mathrm{g}$ per mouse) of antiIFN- $\gamma$ on days $4,5,7,9,10,12$, and 14 after tumor cell implantation. As control for anti-IL-17 or anti-IFN- $\gamma$, antiIL-4, and anti-IL-9, rat IgG1 isotype control (clone HRPN, BioXCell), mouse IgG1 isotype control (clone MOPC21, BioXCell), and mouse IgG2a isotype control (clone C1.18.4, BioXCell) were respectively used.

\section{Adoptive transfer of $\mathrm{T}_{\mathrm{H}} 9$ cells}

B16-OVA tumor-bearing mice ( 5 days after subcutaneous tumor implantation) were injected intravenously with $2 \times 10^{6}$ effector OT-II $\mathrm{T}_{\mathrm{H}} 9$ cells stimulated or not with cGAMP before in vitro polarization. Tumor length and width were measured three times a week using calipers. Mice with tumor size exceeding $300 \mathrm{~mm}^{2}$ or with ulcerated tumors were euthanized in agreement with ethical guidelines.

Alternatively, $2.5 \times 10^{5}$ B16-OVA cells per mouse were injected intravenously in the tail vein. One day after, mice received intravenous injections of $2 \times 10^{6}$ effector OT-II $\mathrm{T}_{\mathrm{H}} 9$ stimulated or not with cGAMP before in vitro polarization. Mice were euthanized 14 days after $\mathrm{T}_{\mathrm{H}} 9$ cell adoptive transfer and lung tumor foci were enumerated in a blinded manner.

B16-F10 tumor bearing mice (7 days after subcutaneous tumor implantation) were first injected intraperitoneally or not with cyclophosphamide (CTX, $200 \mathrm{mg}$ / $\mathrm{kg}$ ) and the next day injected intravenously with $1 \times 10^{6}$ effector Trp1 $\mathrm{T}_{\mathrm{H}} 9$ cells. Trp1 $\mathrm{T}_{\mathrm{H}} 9$ cells were obtained by coculture of naive Trp1 CD 4 T cells with $5 \mu \mathrm{g} / \mathrm{mL}$ of $\operatorname{Trp} 1_{106-133}$ major histocompatibility complex (MHC) class II-restricted TRP1 (SGHNCGTCRPGWRGAACNQK ILTVR) (GenScript)) peptide-loaded $\mathrm{STING}^{-/-}$antigenpresenting cell (APC) (splenocytes depleted from CD4 and CD8 T cells using magnetic enrichment, 1 APC:10 CD4 T-cell ratio) and stimulated or not with cGAMP before in vitro polarization as described further. Tumor length and width were measured three times a week using calipers. Mice with tumor size exceeding $300 \mathrm{~mm}^{2}$ or with ulcerated tumors were euthanized in agreement with ethical guidelines.

\section{Ex vivo procedures}

TIL analysis

MC38 tumors were subjected to mechanic dissociation and enzymatic digestion using the Tumor Dissociation
Kit mouse and the gentleMACS Dissociator (Miltenyi Biotec) according to the manufacturer's instructions. The single-cell suspension was then washed two times using RPMI-1640 supplemented with 10\% FBS, 1\% PSA and $10 \mathrm{mM}$ Hepes (hereafter referred to as complete RPMI). Immune cells were then enriched using CD45 TILs kit (Miltenyi Biotec). Half of the cells were stained with Fixable Viability Dye eFluor 780 (eBioscience), CD45-BV421 (30 F-11, BD Bioscience), and CD4-APC (clone RM4-5, BD Bioscience) for live CD4 T cells sorting using the BD FACSAria-III (BD Biosciences). Sorted CD4 $T$ cells were directly lysed in TriReagent (Ambion) or lysis buffer (Quick-RNA MicroPrep Kit Zymo Research) for RNA analysis.

Remaining cells were then stimulated using Cell Stimulation Cocktail (eBioscience) and Protein Transport Inhibitor Containing Monensin (PTI, eBioscience) diluted in complete RPMI supplemented with $1 \mathrm{mM}$ sodium pyruvate, $2 \mathrm{mM}$ L-glutamine, MEM NEAA (0.1 $\mathrm{mM}$ each AA) and $50 \mu \mathrm{M}$ 2-mercaptoethanol (Gibco)hereafter referred to as mouse T-cell culture mediumfor 2 hours at $37^{\circ} \mathrm{C}$ under $5 \% \mathrm{CO}_{2}$ and subjected to immunostaining and flow cytometry analysis.

As for TIL analysis in MC38-OVA tumor bearing mice, tumor-infiltrating immune cells were restimulated with class II-restricted OVA-peptide (323-339) for 3 hours with Protein Transport Inhibitor Containing Monensin (PTI, eBioscience).

Cell viability was assessed using the LIVE/DEAD Fixable Blue Dead Cell Stain Kit, for UV excitation (Invitrogen) or Fixable Viability Dye eFluor 780 (eBioscience). Extracellular markers were stained with the following antibodies: CD45 APC-Vio770 (clone REA737, Miltenyi Biotec) or CD45-BV421 (clone 30 F-11, BD Bioscience), CD4 PercP-Vio700 (clone REA604, Miltenyi Biotec), and CD8 PE-Vio770 (clone REA601, Miltenyi Biotec). After fixation and permeabilization using the Fixation/Permeabilization Solution Kit (BD Biosciences) according to the manufacturer's instructions, intracellular staining of IFN- $\gamma$ and IL-9 was performed using an anti-mouse IFN- $\gamma$ APC or PE antibody (clone AN.18.17.24 or REA638, Miltenyi Biotec) and mouse IL-9 APC or BV421 antibody (clone RM9A4, BD Bioscience), respectively. Single-cell suspensions were analyzed by flow cytometry using $\mathrm{BD}$ LSR FORTESSA cytometer (BD Biosciences) equipped with BD FACSDiva software (BD Biosciences). Flow cytometry data were then analyzed using Flowlogic software (Miltenyi Biotec). Gating strategies are presented in online supplemental figure S1A.

\section{Mouse primary cell isolation}

Naive CD4 $\mathrm{T}$ cells $\left(\mathrm{CD} 4^{+} \mathrm{CD} 62 \mathrm{~L}^{\mathrm{hi}}\right)$ were isolated from mouse spleen and lymph nodes. CD4 $\mathrm{T}$ cells were first enriched with anti-CD4 microbeads (L3T4) (Miltenyi Biotec) according to the manufacturer's instructions and then further stained with $\mathrm{CD} 4$ Vioblue (clone REA604, Miltenyi Biotec), CD62L PE (clone MEL-14, BD Biosciences) antibodies before sorting using the BD 
FACSAria-III (BD Biosciences). Isolated naive $\mathrm{T}$ cells were routinely $98 \%$ pure. Alternatively, for western blots (WBs) and adoptive transfer experiments, naive CD4 T cells were purified using a naive CD4 T-cell isolation kit (Miltenyi Biotec).

Total effector and memory CD4 T cells from STING V154M/WT mice and their WT littermates were isolated with a mouse $\mathrm{CD} 4^{+}$T-cell isolation kit (Miltenyi Biotec) and directly restimulated using Cell Stimulation Cocktail and PTI diluted in mouse $\mathrm{T}$ cell culture medium for 2 hours at $37^{\circ} \mathrm{C}$ under $5 \% \mathrm{CO}_{2}$ and subjected to immunostaining using LIVE/DEAD Fixable Blue Dead Cell Stain Kit, for UV excitation, CD4 FITC (clone RM4-4, BD Biosciences) or CD4 PercP-Vio700 (clone REA604, Miltenyi Biotec) and CD45 APC-Vio770 (clone REA737, Miltenyi Biotec) antibodies. IFN- $\gamma$ intracellular staining was performed using the Fixation/Permeabilization Solution Kit (BD Biosciences) and IFN- $\gamma$ PE antibody (clone REA638, Miltenyi Biotec). Cells were analyzed using a BD LSRFORTESSA cytometer equipped with BD FACSDiva software and data were analyzed using FlowLogic software. Gating strategies are presented in online supplemental figure S1B.

Total CD8 cells were isolated from $\mathrm{STING}^{-/-}$mouse spleen and lymph nodes using anti-CD8 microbeads (Ly-2) (Miltenyi Biotec) according to the manufacturer's instructions.

\section{Human T-cell isolation}

Human naive CD4 T cells $\left(\mathrm{CD} 4^{+} \mathrm{CD}_{4} 5 \mathrm{RA}^{+}\right)$were isolated from healthy donor blood. CD4 $\mathrm{T}$ cells were purified using RosetteSep Human CD4 ${ }^{+}$T Cell Enrichment Cocktail (StemCell) according to the manufacturer's instructions and then further stained with CD4 BV605 (clone RPA-T4, BioLegend) and CD45RA PE/APC (clone HI100, BioLegend) antibodies before sorting using the BD FACSAria-III.

\section{Cell transfection and treatments}

Mouse naive CD4 $\mathrm{T}$ cells were seeded on plate-bound anti-CD3 (2 $\mu \mathrm{g} / \mathrm{mL}$, clone 17A2; BioXCell) and antiCD28 (2 $\mu \mathrm{g} / \mathrm{mL}$, clone PV1; BioXCell). Human naive CD4 $\mathrm{T}$ cells were seeded on plate-bound anti-CD3 (5 $\mu \mathrm{g} / \mathrm{mL}$, clone OKT-3; BioXCell) and anti-CD28 (2 $\mu \mathrm{g} /$ $\mathrm{mL}$, clone CD28.2; BioLegend). Cells $\left(3 \times 10^{6}\right.$ cells $\left./ \mathrm{mL}\right)$ were transfected for 6 hours with $80 \mu \mathrm{g} / \mathrm{L}$ cyclic dinucleotides (unless specified otherwise) using Opti-MEM Glutamax (Gibco) and Lipofectamine 2000 (Invitrogen), according to the manufacturer's instructions. Alternatively, $1.5 \times 10^{6}$ cells $/ \mathrm{mL}$ CD4 $\mathrm{T}$ cells were treated with indicated doses of 5,6-dimethylxanthenone-4-acetic acid (DMXAA) for 4 hours in mouse T-cell culture medium.

$2^{\prime} 3^{\prime}$-cGAMP (cyclic $\left.\left(\mathrm{G}\left(2^{\prime}, 5^{\prime}\right) \mathrm{pA}\left(3^{\prime}, 5^{\prime}\right) \mathrm{p}\right)\right)$, referred to as cGAMP; $2^{\prime} 3^{\prime}$-cGAMP control (2' $5^{\prime}$-GpAp), referred to as control; as well as DMXAA (Murine STING ligand, Xanthenone Analog) were purchased from InvivoGen.
When indicated, mTOR inhibition was achieved by adding $10 \mathrm{nM}$ of rapamycin (Rapa; Calbiochem, Merck) during the 6-hour transfection step.

\section{T-cell in vitro polarization}

After cell transfection and/or treatment, supernatants were replaced by mouse T-cell culture medium or AIM V medium (Gibco) for human cells containing polarization cytokines as follows: anti-mouse IFN- $\gamma(50 \mu \mathrm{g} / \mathrm{mL}$, clone XMG 1.2; BioXCell) and anti-mouse IL-4 $(50 \mu \mathrm{g} / \mathrm{mL}$, clone 11B11; BioXCell) for mouse $\mathrm{T}_{\mathrm{H}} \mathrm{O}$ cells; IL-12 (10 $\mathrm{ng} / \mathrm{mL}$, Miltenyi Biotec) and anti-mouse IL-4 for mouse $\mathrm{T}_{\mathrm{H}} 1$ cells; TGF- $\beta$ (2 ng/mL, Miltenyi Biotec), IL-4 (20 ng/ $\mathrm{mL}$, Miltenyi Biotec), and anti-mouse IFN- $\gamma$ for mouse $\mathrm{T}_{\mathrm{H}} 9$ cells; IL-6 (20 ng/mL, Miltenyi Biotec), TGF- $\beta$, antimouse IFN- $\gamma$, and anti-mouse IL-4 for mouse $\mathrm{T}_{\mathrm{H}} 17$, IL-12 $(10 \mathrm{ng} / \mathrm{mL}, \mathrm{R} \& \mathrm{D}$ System) and anti-human IL-4 (3.5 $\mu \mathrm{g} /$ $\mathrm{mL}$, clone MP4-25D2; BioXCell) for human $\mathrm{T}_{\mathrm{H}} 1$ cells, TGF- $\beta$ ( $5 \mathrm{ng} / \mathrm{mL}$, Miltenyi Biotec), IL-4 (10 ng/mL, R\&D System), and anti-human IFN- $\gamma(3.5 \mu \mathrm{g} / \mathrm{mL}$, clone NIB42; BioLegend) for human $\mathrm{T}_{\mathrm{H}} 9$ cells. Unless specified otherwise, cells were cultured for 3 days at $37^{\circ} \mathrm{C}$ under $5 \% \mathrm{CO}_{2}$.

\section{IFN- $\gamma$, IL-17, and IL-9 intracellular level measurement}

$\mathrm{T}_{\mathrm{H}} 1$ and $\mathrm{T}_{\mathrm{H}} 17$ cells were restimulated using Cell Stimulation Cocktail and PTI diluted in mouse T-cell culture medium for 2 hours at $37^{\circ} \mathrm{C}$ under $5 \% \mathrm{CO}_{2}$. All cells were stained with Fixable Viability Dye eFluor 780 (eBioscience) or LIVE/DEAD Fixable Blue Dead Cell Stain Kit, for UV excitation and CD4 Vioblue (clone REA604, Miltenyi Biotec). IL-9-GFP expression in $\mathrm{T}_{\mathrm{H}} 9$ cells was assessed by flow cytometry directly after extracellular staining. As for $\mathrm{T}_{\mathrm{H}} 1$ and $\mathrm{T}_{\mathrm{H}} 17$ cells, IFN- $\gamma$ and IL-17 intracellular staining was performed using the Fixation/ Permeabilization Solution Kit (BD Biosciences) and antiIFN- $\gamma$ APC antibody (clone AN.18.17.24, Miltenyi Biotec) or anti-IL-17 BV605 (clone TC11-18H10, BD Bioscience), respectively. Cells were analyzed using a BD FACSCANTO cytometer or a BD LSRFORTESSA cytometer equipped with BD FACSDiva software, and data were analyzed using FlowLogic software. Gating strategies are presented in online supplemental figure S1C-E.

\section{Real-time quantitative PCR}

Total RNA was extracted from $\mathrm{T}$ cells with TriReagent (Ambion) or Quick-RNA MicroPrep Kit (Zymo Research). Depending on experiments, 10-500 ng of total RNA was retrotranscribed using M-MLV Reverse Transcriptase (Invitrogen) or with iScript cDNA Synthesis Kit (Bio-Rad). cDNA was analyzed by real-time quantitative PCR with Power SYBR Green PCR Master Mix or PowerUp SYBR Green Master Mix (Applied Biosystems) or iTaq Universal SYBR Green Supermix 5000 (Bio-Rad) according to the manufacturer's instructions using the ViiA 7 Real-Time PCR System (Applied Biosystems) combined with $500 \mathrm{nM}$ of forward and reverse primers (primer sequences are indicated in online supplemental table S1). 
Gene expression in CD4 T cells isolated from STING V154M/WT mice and MC38 TILs was analyzed using the TaqMan Fast Advanced Master Mix (Applied Biosystems) and TaqMan Gene Expression Assay (Applied Biosystems) according to the manufacturer's instructions. A list of TaqMan assays is depicted in online supplemental table $\mathrm{S} 1$. As for gene expression in CD4 T cells isolated from MC38 TILs, equivalent of $6 \mathrm{ng}$ of cDNA were preamplified with TaqMan PreAmp Master Mix (Applied Biosystems) combined with pooled TaqMan Gene Expression Assays in a final volume of $25 \mu \mathrm{L}$ and according to the manufacturer's instructions.

Expression of target genes was normalized to the expression of mouse Actb or human ACTB (relative expression $(\mathrm{RE})$ ). When indicated, the fold change (FC) in RE was calculated by normalizing data to control conditions (WT cells treated with control, for each time point). When Rapa was used, FCs were calculated by normalizing data to each control condition of Rapa or vehicle treatment.

\section{Cytokine measurement}

Cell culture supernatants were analyzed by ELISA for mouse IFN- $\gamma$ (BD Bioscience), IL-9 (BioLegend), IL-17 (BioLegend) or IFN- $\beta$ (PBL Assay Science) or for human IFN- $\gamma$ (BioLegend) or human IL-9 (BioLegend) according to the manufacturer's instructions.

\section{Western blot}

Total protein extracts were obtained by lysing CD4 T cells into ice-cold RIPA Buffer (Pierce) containing Protease Inhibitor Cocktail (Sigma) and Phosphatase Inhibitor Cocktail 3 (Sigma) on ice for $10 \mathrm{~min}$. Lysates were centrifuged at $13,000 \mathrm{~g}$ at $4^{\circ} \mathrm{C}$ for $10 \mathrm{~min}$, and supernatants were retrieved for WB analyses. For subcellular fractionation, cytosolic (Cyt) and nuclear (Nuc) fractions were purified using the ProteoExtract Subcellular Proteome Extraction Kit (Merk) according to manufacturer's instructions. Protein lysates were separated on 4\%-15\% gradient precast gels (Bio-Rad) before transfer on polyvinylidene difluoride membranes using a MIXED MW program on the Trans-Blot Turbo Transfer System (Biorad). Membranes were blocked in tris-buffered saline (TBS) containing $0.1 \%$ Tween 20 (TBS-T) and supplemented with $5 \%$ bovine serum albumin (BSA) or non-fat dry milk for 1 hour at room temperature and incubated overnight at $4^{\circ} \mathrm{C}$ with primary antibodies diluted in the appropriate blocking buffer. They were then incubated with a horseradish peroxidase-conjugated secondary antibody (Cell Signaling Technology (CST), 7074S) diluted 1:5000 in TBS-T 5\% BSA and proteins were detected using enhanced chemiluminescence (Clarity Western ECL Substrate, Bio-Rad or SuperSignal West Femto Maximum Sensitivity Substrate, ThermoFisher). Densitometry analysis was performed using Image J software.

The following antibodies were purchased from Cell Signaling Technology (CST) and diluted 1:1000 in TBS-T 5\% BSA: Phospho-STING (Ser 366, 19781), TBK1/ NAK (clone D1B4, 3504) Phopho-TBK1 (Ser 172, clone
D52C2, 5483), Phospho-IRF-3 (Ser 396, clone 4D4G, 4947), IRF3 (clone D83B9, 4302), Phospho-NF kappa B P65 (Ser 536, clone 93H1, 3033), NF kappa B P65 (clone D14E12, 8242), Phospho-p70S6K (Thr389, clone 108D2, 9234), and Phospho-S6 (Ser235/236, clone D57.2.2E, 4858). Antibody against histone H3 (clone D1H2, 4499, CST) was diluted 1:2000 in TBS-T 5\% milk. Antibodies against $\beta$-actin horseradish peroxidase (HRP) conjugate (clone 8H10D10, 12262, CST) and anti-GAPDH HRP conjugate (clone 14C10, 3683, CST) were diluted 1:5000 in TBS-T 5\% BSA. Antibody against TMEM173 (19 851-1AP, Proteintech) was diluted 1:1000 in TBS-T 5\% milk.

\section{RNAseq}

STING V154M/WT mouse CD4 T-cell RNAseq analyses

Mouse splenocytes from STING V154M/WT mice $(n=5)$ and control littermates $(n=5)$ were stained with antiCD3-FITC (clone 145-2 C11, BD Biosciences), antiCD4-AF700 (clone RM4-5, BD Biosciences), and DAPI (Sigma) before positive sorting of live $\mathrm{CD}^{+} \mathrm{CD}^{+} \mathrm{T}$ cells using a BD FACSAria Fusion cell sorter (IGBMC Flow Cytometry Facility, Strasbourg, France). Isolated cells were at least $95 \%$ pure. Total RNA was extracted using the RNeasy Plus Micro Kit (Qiagen) according to the manufacturer's instructions. RNAseq analyses were performed by the Genomax Facility (INSERM U1109, ImmunoRhumatologie Moléculaire, Université de Strasbourg, France). Libraries were prepared from $10 \mathrm{ng}$ RNA using SMARTer Stranded Total RNA-Seq Kit, Pico Input Mammalian (Takara) following the manufacturers' instructions. Briefly, random primers were used for firststrand synthesis, and ribosomal cDNA was cleaved by ZapR $\mathrm{V} .2$ in the presence of mammalian R-probes V.2. Libraries were pooled and sequenced (paired-end $2 \times 75 \mathrm{bp}$ ) on a NextSeq500 using the NextSeq 500/550 High Output Kit V.2 according to the manufacturer's instructions (Illumina). For each sample, quality control was carried out and assessed with the next-generation sequencing (NGS) Core Tools FastQC. Reads were aligned against Mus musculus mm10 reference genome using TopHat 2 Aligner, ${ }^{30}$ and gene expression levels were estimated using Cufflinks V.2.1.1. ${ }^{31}$ Differential expression analysis was performed with Cuffdiff V.2.1.1 after exclusion of fragments per kilobase million below 9 .

\section{cGAMP-stimulated mouse $T_{H} 1$ and $T_{H} 9$ cell RNAseq analyses}

Mouse naive CD4 $\mathrm{T}$ cells were isolated and treated with cGAMP or control and polarized in vitro into $\mathrm{T}_{\mathrm{H}} 1$ or $\mathrm{T}_{\mathrm{H}} 9$ cells as described previously for 16 and 48 hours. Total RNA was extracted with TriReagent according to the manufacturer's instructions. Libraries were prepared from $500 \mathrm{ng}$ of total RNA using TruSeq Stranded Total RNA Library Prep kit (Illumina) after rRNA removal with Ribo-zero rRNA Removal Kit (Illumina) following the manufacturers' instructions. RNA sequencing was performed on NextSeq500 device (Illumina). The RNAseq libraries were sequenced with single-end 76 bp reads. Raw FASTQ files were trimmed for residual adapter 
sequences and quality filtered through Trimmomatic software. ${ }^{32}$ Reads were pseudo-aligned against $\mathrm{mm} 10$ genome through Kallisto software and differential expression analysis was performed with DESeq2 R package. ${ }^{33}$ Unsupervised hierarchical clustering of genes was performed by using Clustvis web tool using Ward correlation. Gene set enrichment analysis (GSEA) was performed using GSEA Software ${ }^{3435}$ and using EnrichR web tool ${ }^{36} 37$ with GO Biological Process 2018 database. Data are deposited in the Gene Expression Omnibus database under accession number GSE147300.

\section{Statistical analysis}

Statistical analyses were performed using Prism software (Graph Pad software, La Jolla, California, USA). For twogroup comparisons, Student t-tests was used. For multiple group comparison, ordinary one-way analysis of variance (ANOVA) with Dunnett's multiple comparisons test or ordinary two-way ANOVA with Sidak's multiple comparisons test was used. For survival curve statistics, log-rank (Mantel-Cox) test was used. All $\mathrm{p}$ values are two tailed.

\section{RESULTS \\ STING activation enhances CD4 T-cell effector functions in vivo}

We investigated the immune-related mechanisms that contribute to the anticancer efficacy of cGAMP in vivo. For this, we first relied on the mouse colon cancer model MC38 where we found that intratumoral injection of cGAMP to tumor-bearing mice induced tumor growth control in a STING-dependent manner (online supplemental figure S2A).

Analysis of effector cytokines Ifng, Il9, Ill7a and $I l 4$ mRNA in tumor-infiltrating immune cells revealed that Ifng and Il9, but not $I l 17 a$ or Il4, expression was increased following cGAMP administration (online supplemental figure S2B). We and others showed that CD4 $\mathrm{T}$ cells support the induction of CD8 $\mathrm{T}$ cell-dependent anticancer immune responses, ${ }^{1538}$ and recent studies suggested that CD4 $\mathrm{T}$ cells could contribute to the antitumor properties of STING ligands. ${ }^{39}$ To test this, we have crossed Rag2 ${ }^{-/-}$mice to STING-deficient mice and reconstituted them with either WT or STING-deficient CD4 $\mathrm{T}$ cells as well as with STING-deficient CD8 T cells. We subsequently engrafted MC38 tumor cells to these mice and treated them with cGAMP (figure 1A,B). cGAMP treatment was only effective in mice reconstituted with WT CD4 $\mathrm{T}$ cells, indicating that CD4 $\mathrm{T}$ cells can be directly affected by STING agonists in vivo in the absence of STING-expressing APCs (figure 1A,B), further underscoring the essential contribution of CD4 $\mathrm{T}$ cells in the anticancer efficacy of cGAMP. We then analyzed tumorinfiltrating CD4 T cells and found an increased expression of interferon-beta (Ifnb1) and interferon-stimulated gene (ISG) transcripts (Ccl5, Ifit1, and Mx2) in cells isolated from mice treated with cGAMP compared with controls (online supplemental figure S2C). Interestingly, we also found that cGAMP increased IFN- $\gamma$ production from tumor-infiltrating CD4 T cells (figure 1C,D) as well as from MC38-OVA tumor-infiltrating OVA-specific CD4 T cells (online supplemental figure S2D), suggesting that STING activation enhances CD4 T-cell effector functions in vivo. To test this, we evaluated the impact of the gainof-function V154M mutation of STING, which drives its constitutive activation, ${ }^{29}$ on $\mathrm{CD} 4 \mathrm{~T}$ cell-derived IFN- $\gamma$ secretion. Effector CD4 $\mathrm{T}$ cells isolated from STING V154M/WT mouse spleens featured increased IFN- $\gamma$ production on ex vivo restimulation than those from control littermates (figure 1E). Transcriptional analysis of CD4 T cells isolated from STING V154M/WT mice by RNAseq revealed an upregulation of $\mathrm{T}_{\mathrm{H}} 1$-related genes compared with control littermates (online supplemental figure S2E). We then tested the contribution of IFN- $\gamma$ to the anticancer activity of cGAMP in vivo. In the immunogenic MC38 model, we found that IFN- $\gamma$ neutralization impaired cGAMP antitumor effect as shown by the reduction of both cGAMP-mediated tumor growth control and mouse survival (figure 1F). When we performed a similar experiment in mice bearing poorly immunogenic B16-F10 tumors, we noted that the beneficial anticancer of cGAMP was abrogated by IFN- $\gamma$ blockade (figure 1G). The latter observation is in line with the previously reported key contribution of IFN- $\gamma$ in preventing B16 tumor growth in vivo. ${ }^{40}$ To further test whether IFN- $\gamma$ is important for the anticancer effects of cGAMP mediated by $\mathrm{CD} 4 \mathrm{~T}$ cells, we have repeated our adoptive transfer model experiments presented in figure 1A upon neutralization of IFN- $\gamma$. Rag2 ${ }^{-/-} \mathrm{STING}^{-/-}$mice reconstituted with WT CD4 T cells and injected with anti-IFN- $\gamma$ antibody have a markedly reduced response to cGAMP compared with mice injected with isotype control, demonstrating that IFN- $\gamma$ is a key contributor to the anticancer effects of cGAMP mediated by CD4 T cells in vivo (online supplemental figure S2F). IFN- $\gamma$ neutralization does not fully abrogate the CD4 $\mathrm{T}$ cell-mediated anticancer effects of cGAMP in vivo, suggesting that other effector cytokines could also contribute to the anticancer effect of cGAMP. Because our analyses of tumor-bearing mice treated with cGAMP also revealed an increased frequency of IL-9producing CD4 $\mathrm{T}$ cells in the tumor microenvironment (online supplemental figure $\mathrm{S} 2 \mathrm{G}, \mathrm{H}$ ), we finally evaluated the contribution of other $\mathrm{CD} 4 \mathrm{~T}$ cell-derived cytokines (IL-9, IL-17, and IL-4) to the anticancer effects of cGAMP. While IL-9-neutralizing antibodies reduced the anticancer activity of cGAMP in both MC38 and B16-F10 tumor models (figure 1H), neutralization of IL-4 and IL-17 had no effect on cGAMP-mediated tumor growth control (figure 1I,J). Overall, these results indicate that STING activation enhances CD4 T-cell effector functions and reveal that IFN- $\gamma$ and IL-9 contribute to the antitumor activity of cGAMP in vivo.

STING ligands enhance $T_{H} 1$ and $T_{H} 9$ cell differentiation in vitro Because of the key contribution of IFN- $\gamma$ and IL-9 to the anticancer effects induced by cGAMP in vivo, we next assessed 


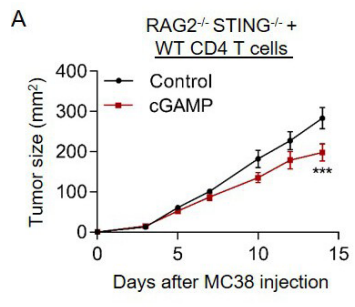

C
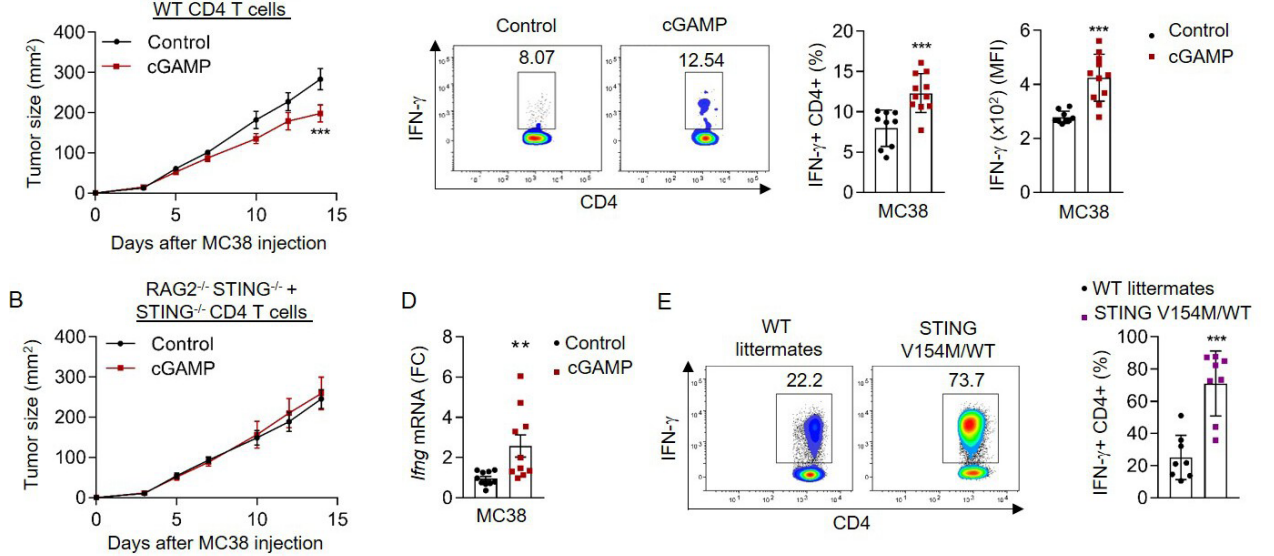

$\mathrm{F}$
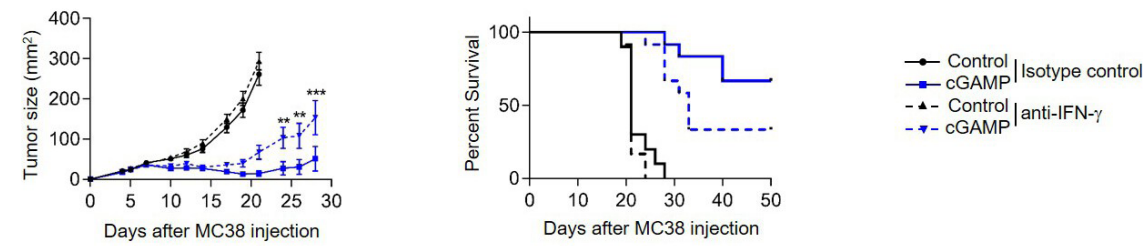

-1 - Contro

$\rightarrow$ - Control $\mid$ anti-IFN- $\gamma$
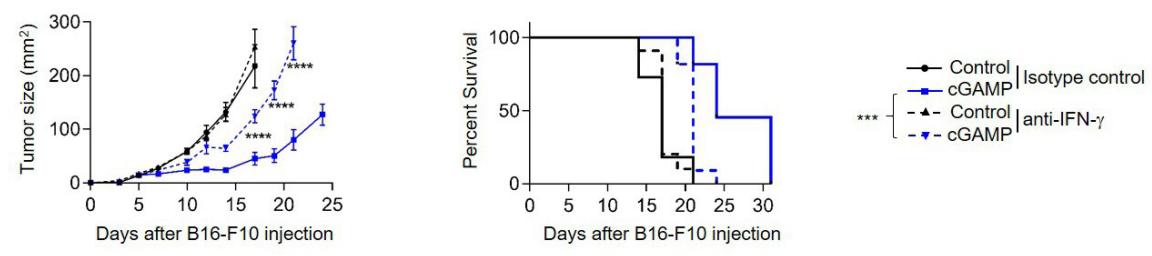

$\mathrm{H}$
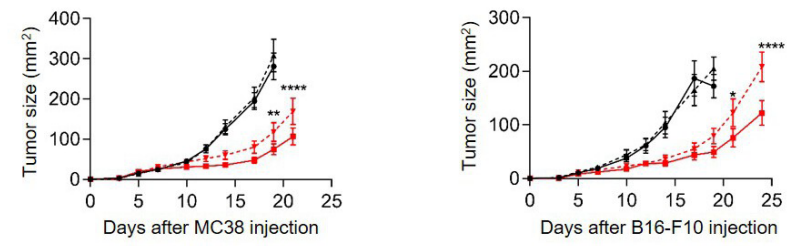

$\rightarrow$ Control | Isotype control

$\rightarrow-$ - Control

- - - cGAMP - Conti-IL-9

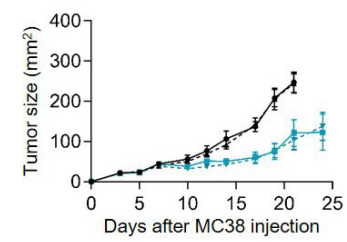

$J$

$\rightarrow$ Control | Isotype control
- - cGAMP

$-\downarrow-$ Control $\mid$ anti-IL-17

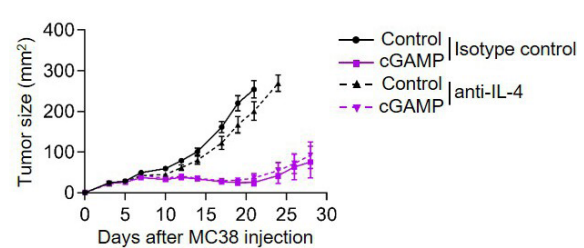

Figure 1 STING activation enhances CD4 T-cell effector functions in vivo. (A,B) MC38 tumor size in STING ${ }^{-/}$RAG2 ${ }^{-/-}$mice reconstituted with WT (A) or STING ${ }^{-/-}$(B) CD4 T cells and STING ${ }^{-/-}$CD8 T cells, and treated or not intratumorally with cGAMP. Mean \pm SEM of $n=8-9$ mice per group pooled from two independent experiments. (C,D) TIL analysis from MC38 tumor-bearing WT mice after intratumoral cGAMP treatment. (C) IFN- $\gamma$ production (representative plots (left); frequency (middle) and MFI (right)). (D) Ifng mRNA expression (FC) from FACS-sorted CD4 T cells. Mean \pm SD from three independent experiments where each dot represents one mouse ( $\mathrm{n}=9-11$ mice). (E) IFN- $\gamma$ production (representative plots (left) and frequency (right)) in splenic CD4 T cells isolated from STING V154M/WT mice or WT littermates. Mean \pm SD from three independent experiments where each dot represents one mouse ( $n=8$ mice). $(F, G)$ Tumor size (left) and survival (right) in MC38 (F) and B16-F10 (G) tumor-bearing mice treated or not intratumorally with cGAMP as well as intraperitoneally with anti-IFN- $\gamma$ or its control IgG. Mean \pm SEM of $n=10-12$ $(\mathrm{F})$ and $\mathrm{n}=11(\mathrm{G})$ mice per group pooled from two independent experiments. $(\mathrm{H})$ Tumor size in MC38 (left) and B16-F10 (right) tumor-bearing mice treated or not intratumorally with cGAMP as well as intraperitoneally with anti-IL-9 antibodies or its control IgG. Mean \pm SEM of $n=11$ (left) and $n=8-10$ (right) mice per group pooled from two independent experiments. (I,J) Tumor size in MC38 tumor-bearing mice treated or not intratumorally with cGAMP as well as intraperitoneally with anti-IL-17 (I) or antiIL-4 (J) antibodies or their respective control IgG. Mean \pm SEM of $n=11-12(\mathrm{I})$ and $n=10-11(\mathrm{~J})$ mice per group pooled from two independent experiments. $P$ values $\left({ }^{*} p<0.05,{ }^{* *} p<0.01,{ }^{* \star *} p<0.001,{ }^{* \star *} p<0.0001\right)$ determined by unpaired t-test (C-E), Twoway analysis of variance (A,B,F-J) or log-rank test (F,G, right; survival only). cGAMP, 2'3'-cyclic guanosine monophosphateadenosine monophosphate; FC, fold change; IFN- $\gamma$, interferon gamma; IL, interleukin; MFI, mean fluorescence intensity; STING, stimulator of interferon genes; TIL, tumor-infiltrating lymphocyte; WT, wild type. 
A

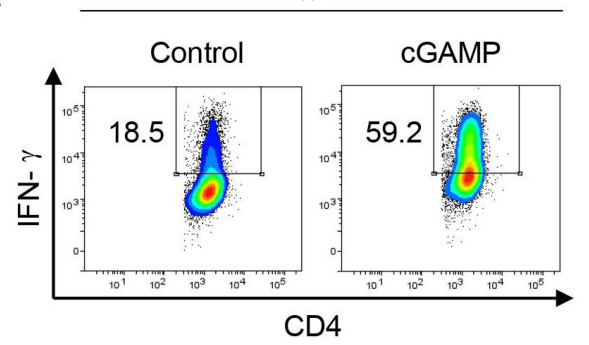

C

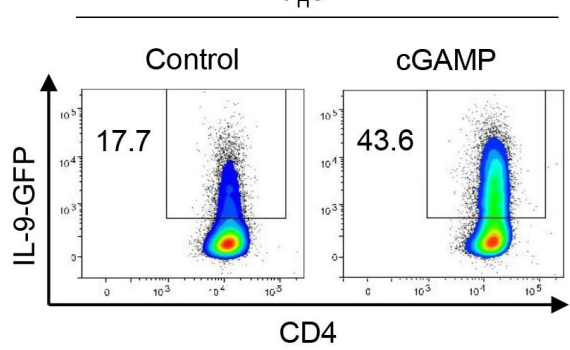

E

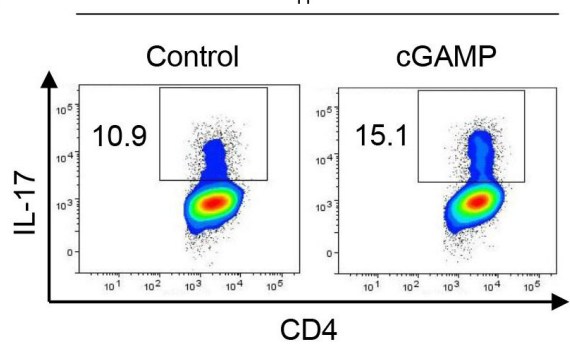

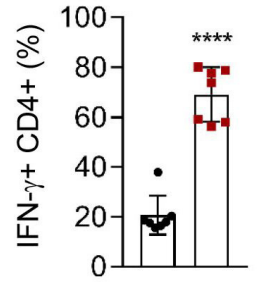

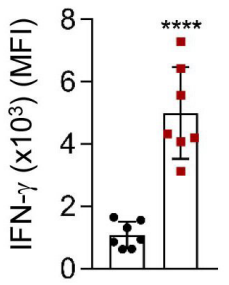

B
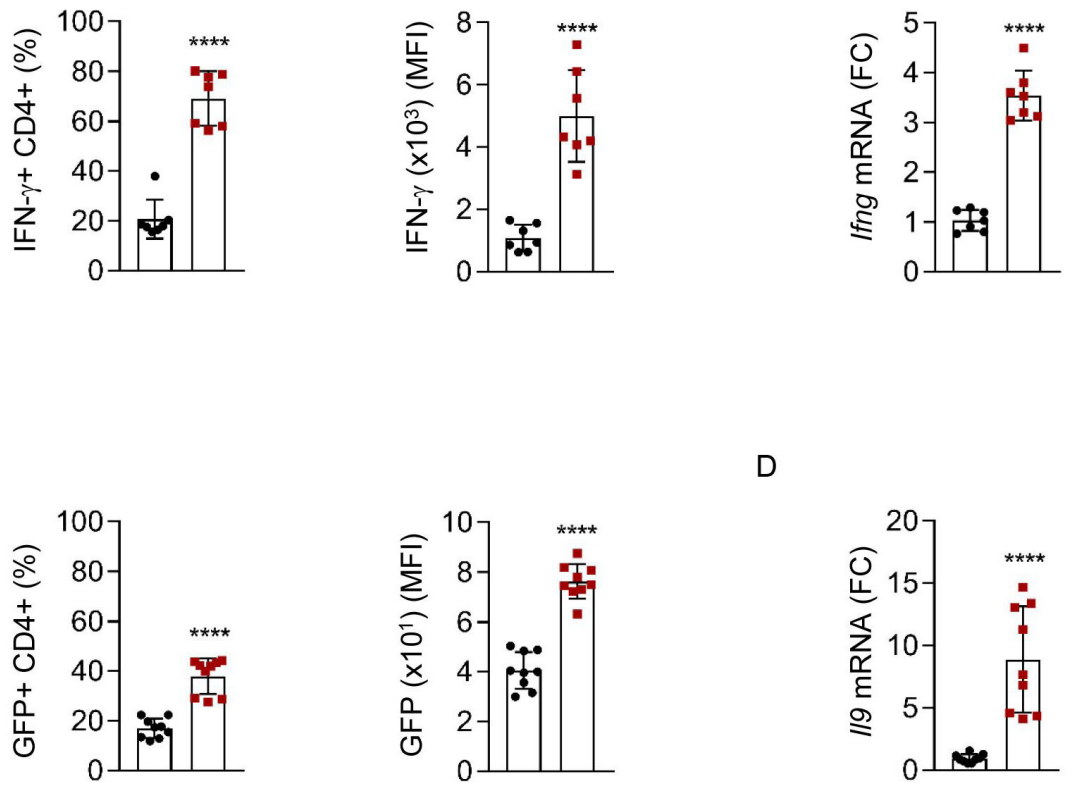

D

F

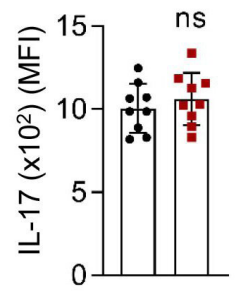

Figure 2 cGAMP enhances $T_{H} 1$ and $T_{H} 9$ cell differentiation in vitro. (A,B) IFN- $\gamma$ production (representative plots (A, left); frequency ( $A$, middle) and MFI (A, right) and mRNA expression (FC), (B) from WT naive CD4 T cells stimulated with cGAMP and polarized into $T_{H} 1$ cells. (C,D) IL-9 production (representative plots $(C$, left); frequency $(C$, middle) and $M F I(C$, right) and mRNA expression (FC), (D) from IL-9-GFP naive CD4 T cells stimulated with cGAMP and polarized into $T_{H} 9$ cells. (E,F) IL-17 production (representative plots (E, left); frequency (E, middle) and MFI (E, middle) and mRNA expression (FC) (F) from WT naive CD4 T cells stimulated with cGAMP and polarized into $T_{H} 17$ cells. Mean $\pm S D$ of replicates pooled from three independent experiments. $P$ values $\left({ }^{* \star *} p<0.001,{ }^{* \star * \star} p<0.0001\right)$ determined by unpaired t-tests. cGAMP, $2^{\prime} 3^{\prime}$-cyclic guanosine monophosphate-adenosine monophosphate; FC, fold change; GFP, green fluorescent protein; IFN- $\gamma$, interferon gamma; IL, interleukin; MFI, mean fluorescence intensity; ns, not significant.

whether STING signaling directly affects the differentiation of CD4 T cells in vitro. For this, we tested if cGAMP or negative control dinucleotide (control), which does not activate STING signaling, could modulate the polarization of naive CD4 $\mathrm{T}$ cells into $\mathrm{T}_{\mathrm{H}} 1, \mathrm{~T}_{\mathrm{H}} 9$ or $\mathrm{T}_{\mathrm{H}} 17$ cells in the absence of APCs. In line with our in vivo data, naive CD4 T cells activated with anti-CD3 and anti-CD28 antibodies in the presence of cGAMP, and polarized into $\mathrm{T}_{\mathrm{H}} 1$ and $\mathrm{T}_{\mathrm{H}} 9$ cells, respectively, featured enhanced IFN- $\gamma$ and IL-9 expression and secretion 3 days after differentiation initiation compared with control (figure 2A-D). In contrast, we noted that cGAMP featured limited and variable effects on $\mathrm{T}_{\mathrm{H}} 17$ cell differentiation (figure $2 \mathrm{E}, \mathrm{F}$ ). We found that the ability of cGAMP to enhance $T_{H} 1$ and $T_{H} 9$ cell differentiation was lost when CD4 $\mathrm{T}$ cells were isolated from STING-deficient mice $\left(\mathrm{STING}^{-/-}\right)$, underscoring the STING-dependent activity of cGAMP in CD4 $\mathrm{T}$ cells (figure $3 \mathrm{~A}, \mathrm{~B}$ ).

We also tested the ability of increasing doses of cGAMP to affect $\mathrm{T}_{\mathrm{H}} 1$ and $\mathrm{T}_{\mathrm{H}} 9$ cell differentiation. Increasing doses of cGAMP enhanced $T_{H} 1$ and $T_{H} 9$ cell differentiation without significantly affecting cell death at the doses tested (figure 3C,D, and online supplemental figure S3A,B). The mouse STING ligand DMXAA, which is cell-permeable and potently activates STING, was proposed to induce T-cell death when used at the dose of $10 \mu \mathrm{g} / \mathrm{mL} .{ }^{26}$ Here, we found that while low doses of DMXAA enhanced $\mathrm{T}_{\mathrm{H}} 1$ differentiation, higher doses began to concomitantly trigger cell death (figure 3E and online supplemental figure S3C). A higher dose of DMXAA was required to observe enhanced $\mathrm{T}_{\mathrm{H}} 9$ differentiation (figure $3 \mathrm{~F}$ ) in line with the fact that $\mathrm{T}_{\mathrm{H}} 9$ cells 
A

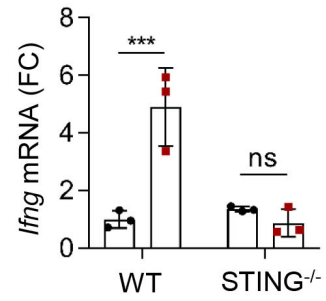

C

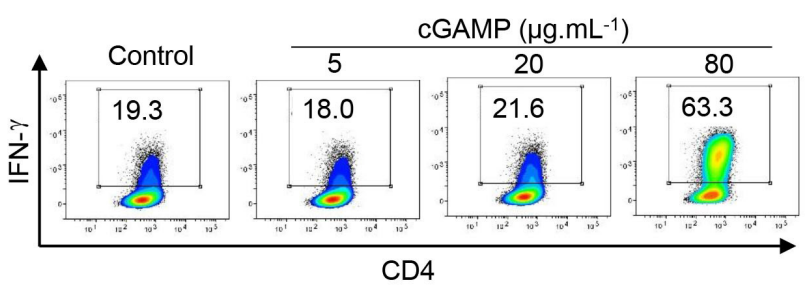

D

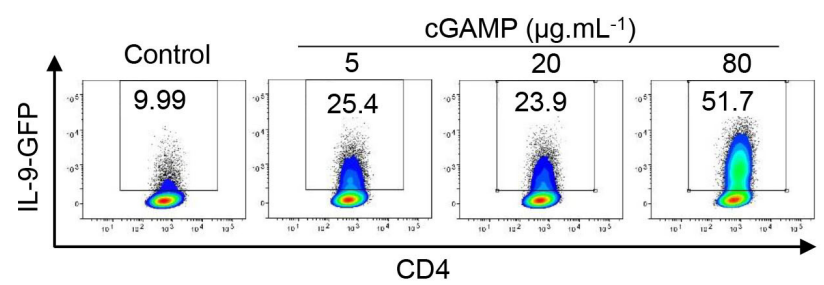

$\mathrm{E}$

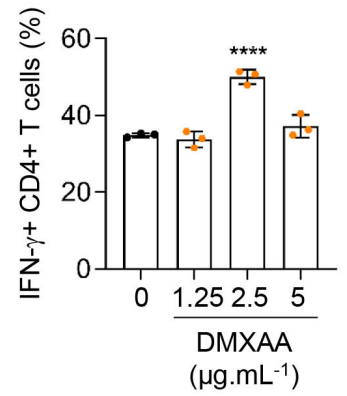

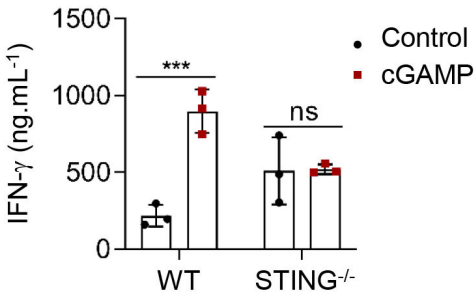

CD4
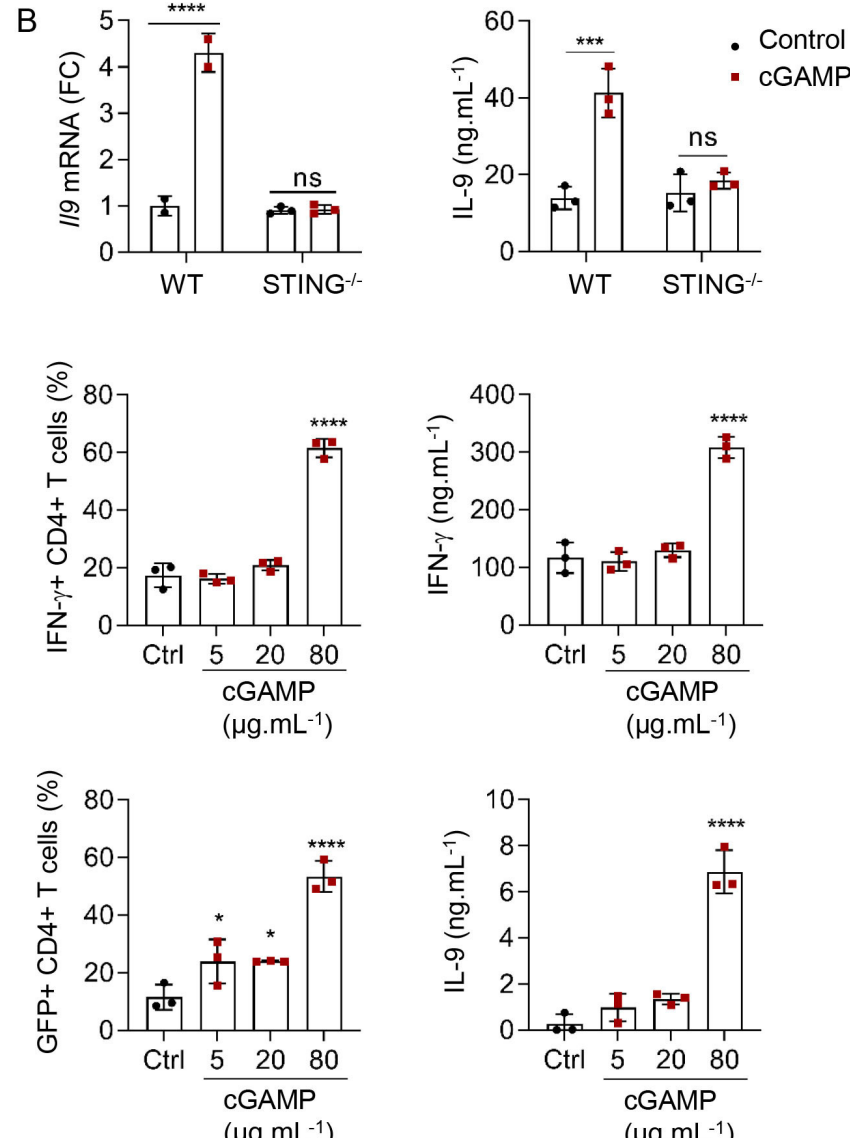

$\mathrm{F}$

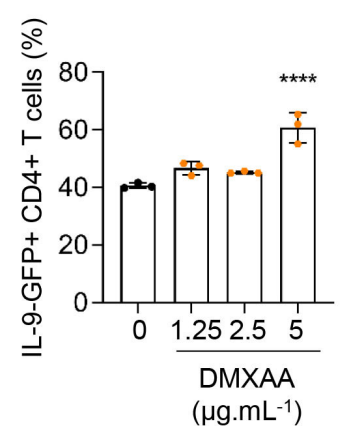

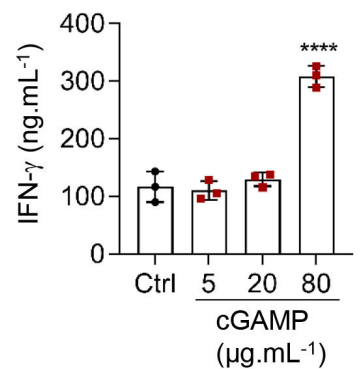
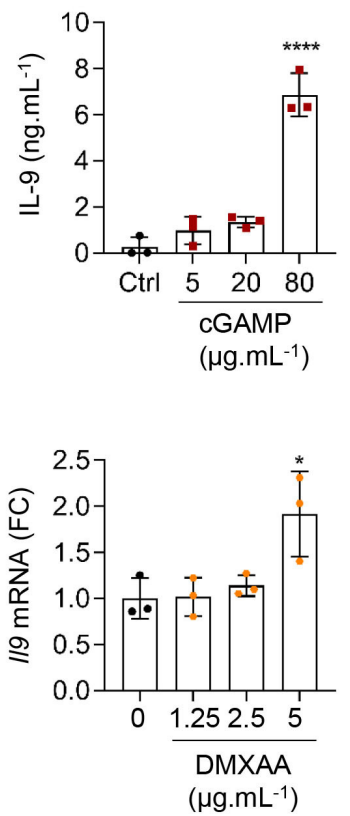

Figure 3 STING ligands enhance $T_{H} 1$ and $T_{H} 9$ cell differentiation in vitro. $(A, B)$ Ifng $(A)$ or II9 (B) mRNA expression (FC, left) and IFN- $\gamma(\mathrm{A})$ or IL-9 (B) secretion (right) from WT or STING ${ }^{-/}$naive CD4 T cells stimulated with cGAMP or control and polarized into $\mathrm{T}_{\mathrm{H}} 1$ (A) or $\mathrm{T}_{\mathrm{H}} 9$ (B) cells. (C,D) IFN- $\gamma$ (C) or IL-9 (D) production (representative plots (left), frequency (middle)) and secretion (right) from WT (C) or IL-9-GFP (D) naive CD4 T cells stimulated with cGAMP or control and polarized into $T_{1} 1$ (C) or $T_{4} 9$ (D) cells.

$(\mathrm{E}, \mathrm{F})$ IFN- $\gamma(\mathrm{E})$ or IL-9 (F) production (frequency, left) and Ifng (A) or II9 (B) mRNA expression (FC, right) from WT (E) or IL-9GFP (F) naive CD4 T cells stimulated with DMXAA or dimethyl sulfoxide (DMSO) and polarized into $T_{H} 1(E)$ or $T_{H} 9$ (F) cells. Mean $\pm S D$ of replicates from one experiment representative of three $(C, D)$ or two $(A, B, E, F)$ experiments. $P$ values $\left({ }^{*} p<0.05,{ }^{* \star *} p<0.001\right.$, $\left.{ }^{* * \star *} \mathrm{p}<0.0001\right)$ determined by two-way ANOVA $(\mathrm{A}, \mathrm{B})$ or one-way ANOVA $(\mathrm{C}-\mathrm{F})$. ANOVA, analysis of variance; cGAMP, $2^{\prime} 3^{\prime}$-cyclic guanosine monophosphate-adenosine monophosphate; FC, fold change; GFP, green fluorescent protein; IFN- $\gamma$, interferon gamma; IL, interleukin; ns, not significant; WT, wild type.

appeared less sensitive to DMXAA-induced cell death than $\mathrm{T}_{\mathrm{H}} 1$ cells (online supplemental figure S3D). While these results are in agreement with previous studies proposing that potent STING activation in T cells triggers cell death, ${ }^{246}$ they also indicate that milder STING activation can enhance T-cell effector properties without killing them, a finding that will be of importance as this pathway is targeted for cancer therapy development. Interestingly, we noted a higher sensitivity of $\mathrm{T}_{\mathrm{H}} 1$ cells to STING-induced death (online supplemental figure S3A-D, right panels), suggesting that the balance between enhancement of effector properties and cell death varies between $\mathrm{CD} 4 \mathrm{~T}$-cell subsets. Altogether, these data show that the strength of STING engagement defines the consequences of STING activation on CD4 T-cell fate.

Overall, our results indicate that ligand-induced STING activation enhances the secretion of effector cytokines IFN- $\gamma$ and IL-9 from $T_{H} 1$ and $T_{H} 9$ cells and thus controls the effector functions of both CD4 T-cell subsets. 


\section{Transcriptional regulation of $\mathrm{T}_{\mathrm{H}} 1$ and $\mathrm{T}_{\mathrm{H}} 9$ cell differentiation} following STING activation

We evaluated whether STING activation affected $T_{H} 1$ and $\mathrm{T}_{\mathrm{H}} 9$ cell differentiation at the transcriptional level. While the expression levels of Ifng and $I l 9$ were strongly enhanced in differentiating $\mathrm{T}_{\mathrm{H}} 1$ and $\mathrm{T}_{\mathrm{H}} 9$ cells on cGAMP treatment, no notable change in the expression of the $\mathrm{T}_{\mathrm{H}} 1$-specific and $\mathrm{T}_{\mathrm{H}}$ 9-specific transcription factors $T b \times 21 /$ Irf1 and Spi1/Irf4/Irf8/Batf, respectively, was detected (figure 4A and online supplemental figure S4A). In line with their enhanced expression of $I l 9, \mathrm{~T}_{\mathrm{H}} 9$ cells treated with cGAMP featured lower expression levels of Foxp $3^{7}$ (figure 4A). No significant induction of Gata3 or Rorc expression in $\mathrm{T}_{\mathrm{H}} 1$ and $\mathrm{T}_{\mathrm{H}} 9$ cells on cGAMP treatment was found, suggesting that cGAMP enhances but does not skew $\mathrm{T}_{\mathrm{H}} 1$ and $\mathrm{T}_{\mathrm{H}} 9$ cell differentiation (figure $4 \mathrm{~A}$ ).

To unravel the mechanisms explaining how STING activation promotes enhanced $T_{H} 1$ and $T_{H} 9$ cell differentiation, we interrogated the intracellular events following activation of STING with cGAMP in mouse CD4 T cells. We found that cGAMP triggered STING and TBK1 phosphorylation as well as Nuc translocation of the phosphorylated forms of p65 and IRF3 (figure 4B,C, and online supplemental figure S4B,C), which are features of STING activation. ${ }^{41}$ In addition, type I IFN (Ifna and Ifnb1), ISG (Ifit1, Ifit2, Mx2 and Cxcl10) and proinflammatory gene (Tnfa and Il6) transcript levels measured in CD4 T cells 6 hours after stimulation with cGAMP were increased (figure 4D and online supplemental figure S4D).

We next performed RNAseq analysis on $\mathrm{T}_{\mathrm{H}} 1$ and $\mathrm{T}_{\mathrm{H}} 9$ differentiated for 16 and 48 hours after stimulation with cGAMP or control. Because we noted cGAMP induced type I IFN and ISG expression in CD4 T cells in vivo (online supplemental figure S2C), we investigated whether cGAMP was intrinsically triggering a global transcriptional program in differentiating $\mathrm{T}_{\mathrm{H}} 1$ and $\mathrm{T}_{\mathrm{H}} 9$ cells that was linked with the induction of a typical type I IFNdriven response. For this, we performed GSEA for type I IFN production (GO:0032479), ISGs and regulation of inflammatory response signatures (GO:0050727). GSEA revealed that these three gene sets were enriched in both $\mathrm{T}_{\mathrm{H}} 1$ and $\mathrm{T}_{\mathrm{H}} 9$ cells stimulated with cGAMP 16 and 48 hours after in vitro polarization (figure $4 \mathrm{E}$ and online supplemental figure S4E). Finally, Trim30a, which was recently identified as a negative feedback regulator of the STING pathway in DCs, ${ }^{42}$ and Usp18, which was shown to be involved in STING deubiquitination and stabilization, ${ }^{43}$ were strongly induced in both $\mathrm{T}_{\mathrm{H}} 1$ and $\mathrm{T}_{\mathrm{H}} 9$ cells stimulated with cGAMP (online supplemental table S2). Gene transcript analyses also revealed that cGAMP triggers a transcriptional program typically related to STING activation, which notably leads to the expression of type I IFN and ISGs. Importantly, this program is maintained over time as reflected by the expression of ISG including Isg 15 , $M x 1, M x 2, C x c l 10$, and $\operatorname{Irf} 7$ that was markedly increased in CD4 T cells stimulated with cGAMP and differentiated into $\mathrm{T}_{\mathrm{H}} 1$ or $\mathrm{T}_{\mathrm{H}} 9$ cells for 16 or 48 hours (figure $4 \mathrm{~F}$ and online supplemental figure S4F). Finally, in line with our previous results, $\mathrm{T}_{\mathrm{H}} 1$ and $\mathrm{T}_{\mathrm{H}} 9$ polarized after cGAMP stimulation featured a marked increase in Ifng and $I l 9$ expression, respectively, but also in other cytokines/ chemokines including Gzmb, $I l 2$ and $C c l 4$ for $\mathrm{T}_{\mathrm{H}} 1$ cells and Il21, Tnfsf4 (OX40L), Tnfsf 8 , as well as $I l 10$ for $\mathrm{T}_{\mathrm{H}} 9$ cells (online supplemental figure $\mathrm{S} 4 \mathrm{G}, \mathrm{H}$ ). These analyses were confirmed by our investigation of the biological pathways engaged in these cells using EnrichR (online supplemental figure S4I).

Collectively, these data reveal that the engagement of STING in differentiating $\mathrm{T}_{\mathrm{H}} 1$ and $\mathrm{T}_{\mathrm{H}} 9$ cells not only enhances the expression of their respective effector cytokines but also engages a transcriptional program driving the expression of type I IFNs and proinflammatory genes.

\section{STING signaling enhances $T_{H} 1$ cell differentiation through IRF3 signaling}

Since we noted a strong induction of a type I IFN transcriptional program in $\mathrm{T}_{\mathrm{H}} 1$ and $\mathrm{T}_{\mathrm{H}} 9$ cells on STING activation, we next tested its relevance in the differentiation of these cells. First, we observed that cGAMP induced not only Ifnb1 transcription but also the secretion of IFN- $\beta$ from differentiating $\mathrm{T}_{\mathrm{H}} 1$ and $\mathrm{T}_{\mathrm{H}} 9$ cells (figure $5 \mathrm{~A}$ ). The induction of type I IFN expression in response to STING activation in myeloid cells has been shown to depend on the transcription factor IRF3. ${ }^{44}{ }^{45}$ We thus tested the contribution of IRF3 in mediating the effects of cGAMP in differentiating $\mathrm{T}_{\mathrm{H}} 1$ and $\mathrm{T}_{\mathrm{H}} 9$ cells. For this, we polarized naive CD4 $\mathrm{T}$ cells from control and Irf3-deficient $\left(\mathrm{IRF}^{-/-}\right.$) mice into $\mathrm{T}_{\mathrm{H}} 1$ and $\mathrm{T}_{\mathrm{H}} 9$ cells. IRF3 contributed to the cGAMP-induced IFN- $\gamma$ production from differentiating $\mathrm{T}_{\mathrm{H}} 1$ cells (figure $5 \mathrm{~B}, \mathrm{C}$ ). However, cGAMP conserved its ability to enhance the differentiation of IRF3-deficient naive $\mathrm{T}$ cells into $\mathrm{T}_{\mathrm{H}} 9$ cells (figure $5 \mathrm{D}$ ). In line with this role for IRF3 in cGAMP-driven $\mathrm{T}_{\mathrm{H}} 1$ differentiation, levels of phosphorylated IRF3 remained increased in the cytosol and the nucleus of $\mathrm{T}_{\mathrm{H}} 1$ cells 16 hours after differentiation initiation (figure $5 \mathrm{E}$ and online supplemental figure $\mathrm{S} 5 \mathrm{~A})$. We then directly assessed the functional consequences of cGAMP-driven type I IFN secretion in differentiating $\mathrm{T}_{\mathrm{H}} 1$ and $\mathrm{T}_{\mathrm{H}} 9$ cells using type I IFN receptor-deficient mice $\left(\right.$ IFNAR $^{-/-}$). We found that $T_{H} 1$ but not $\mathrm{T}_{\mathrm{H}} 9$ cells relied on cGAMP-induced type I IFN receptor-dependent for the enhancement of their differentiation (figure $5 \mathrm{~F}-\mathrm{H}$ ). Overall, these results underscore a key contribution of STING-induced IRF3 activation and type I IFN secretion in the cGAMP-driven enhancement of $\mathrm{T}_{\mathrm{H}} 1$ cell differentiation.

\section{STING signaling enhances $T_{H} 9$ effector functions through activation of $\mathrm{mTOR}$ signaling}

STING engages NF- $\mathrm{KB}$ signaling and the activation of NF- $\kappa B-p 65$ by STING is essential for antiviral immunity. ${ }^{46}$ $\mathrm{NF}-\kappa \mathrm{B}$ (p65) was also proposed to bind the mouse $I l 9$ promoters ${ }^{47}$ and enhance $I l 9$ expression in the context of $\mathrm{T}_{\mathrm{H}} 9$ cell differentiation with proinflammatory factors. ${ }^{9}$ We generated mice conditionally lacking p65 expression in T cells $\left(\mathrm{p} 65^{\mathrm{CD} 4 \mathrm{cre} /+}\right)$ to investigate the ability of STING 

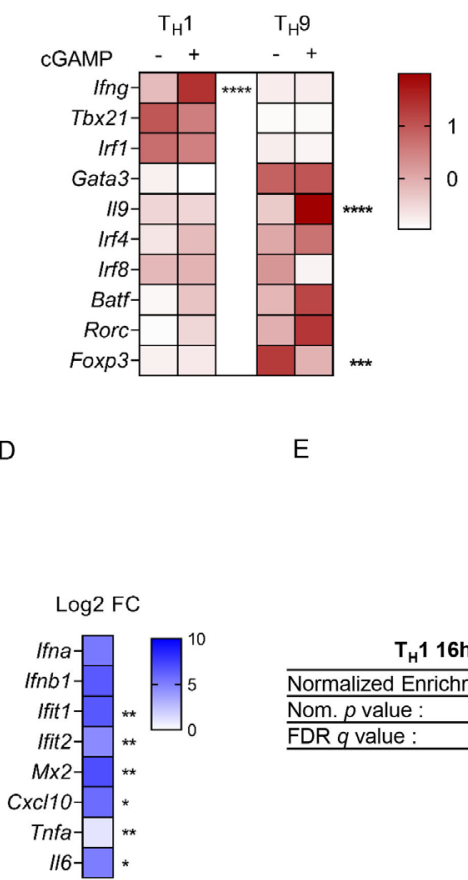

$\mathrm{F}$

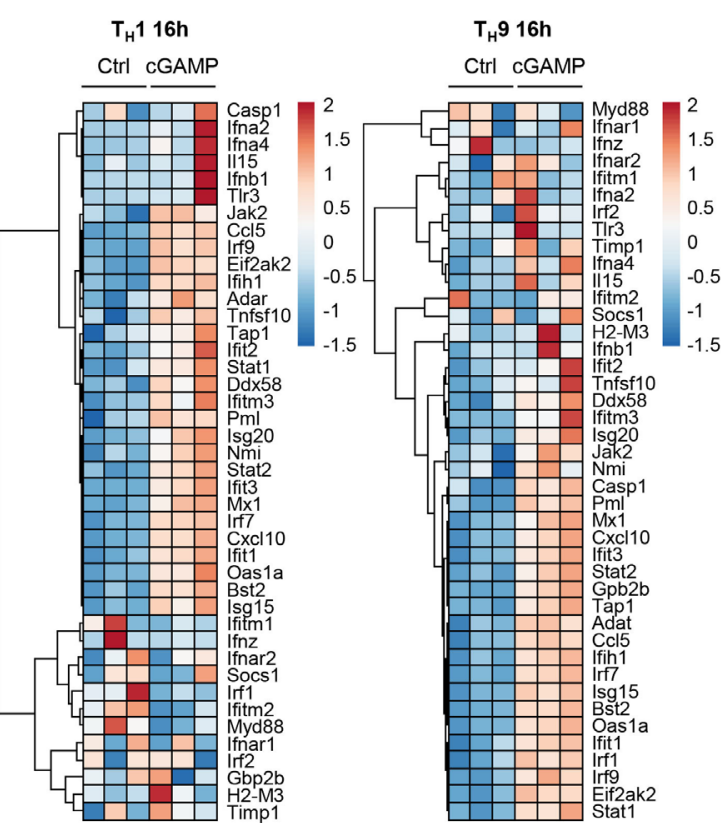

D E CGAMP - $+\mathrm{kDa}$ pSTING (S366) $\longrightarrow 40$ STING $=35$ $A C T I N=45$

CGAMP $-\quad+k D a$
PTBK1 (S172) $\square 84$
TBK1 $\square 84$
ACTIN $\square 45$
cGAMP $\frac{\text { Cyt }}{-+} \frac{\text { Nuc }}{-+}$ kDa p-p65 NF-kB (S536) - 65 p65 NF-kB 65 GAPDH 237 HISTONE H3 $\square 17$

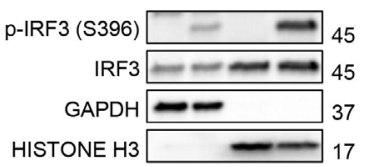

Regulation of Type I Interferon Interferon Stimulated Regulation of inflammatory production (GO:0032479) Genes (ISG) response (GO:0050727)

Figure 4 Transcriptional regulation of $T_{H} 1$ and $T_{H} 9$ cell differentiation following STING activation. (A) Heatmap representing Ifng, Tbx21, IIf1, Gata3, II9,IIf4, Irf8, Batf, Rorc, and Foxp3 mRNA expression (Z-score) from naive CD4 T cells stimulated with cGAMP or control and polarized into $T_{H} 1$ or $T_{H} 9$ cells for 72 hours. (B-D) WT naive CD4 T cells stimulated with cGAMP or control for 4(B) or 6(C,D) hours. (B) STING, p-STING, TBK1 and p-TBK1 protein levels. (C) Cyt and Nuc localization of IRF3, p65 NF-KB and their phosphorylated forms. (D) Heatmap representing Ifna, Ifnb1, II6, Tnfa, Ifit1, Ifit2, Mx2 and Cxc/10 mRNA expression (Log2FC) between CD4 T cells stimulated with cGAMP and control. Mean of replicates from three independent experiments. $P$ values $\left({ }^{*} \mathrm{p}<0.05,{ }^{* *} \mathrm{p}<0.01,{ }^{* * *} \mathrm{p}<0.001,{ }^{* * *} \mathrm{p}<0.0001\right)$ determined by two-way analysis of variance $(A)$ or unpaired $t$ test (D). (E,F) RNA sequencing analysis from WT naive CD4 T cells stimulated with cGAMP or control and polarized into $\mathrm{T}_{\mathrm{H}} 1$ or $\mathrm{T}_{\mathrm{H}} 9$ cells for 16 hours. Biological replicates from three independent experiments. (E) Gene set enrichment analysis comparing expression in cGAMP-stimulated cells to control cells. Enrichment plot and score, Nom. P value and FDR $q$ value shown for the three gene sets. (F) Heatmaps illustrating the hierarchical clustering of expression levels (rld values) of ISGs. cGAMP, 2'3'-cyclic guanosine monophosphate-adenosine monophosphate; Cyt, cytosolic; FDR, false discovery rate; ISG, interferon-stimulated gene; Nom, nominal; Nuc, nuclear; STING, stimulator of interferon genes; WT, wild type. 

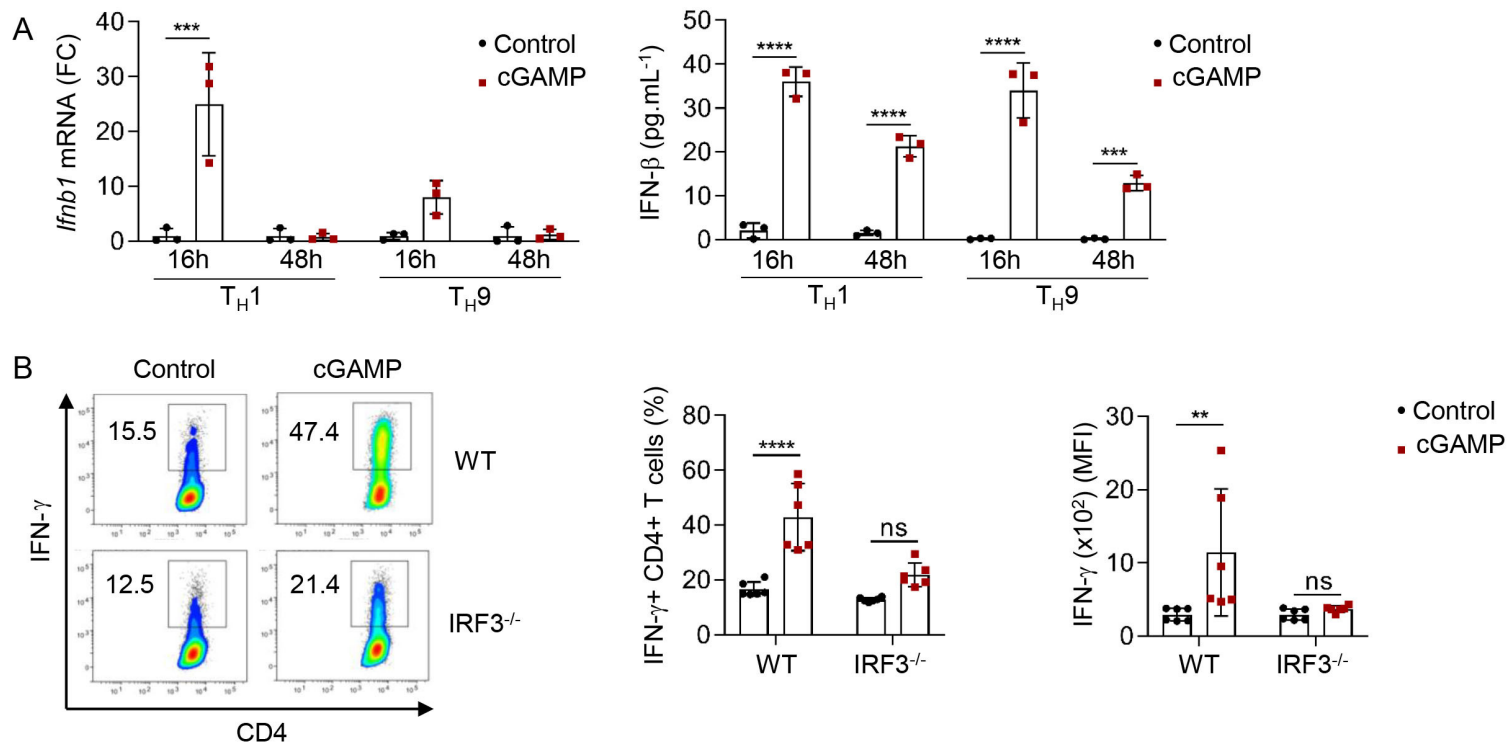

C
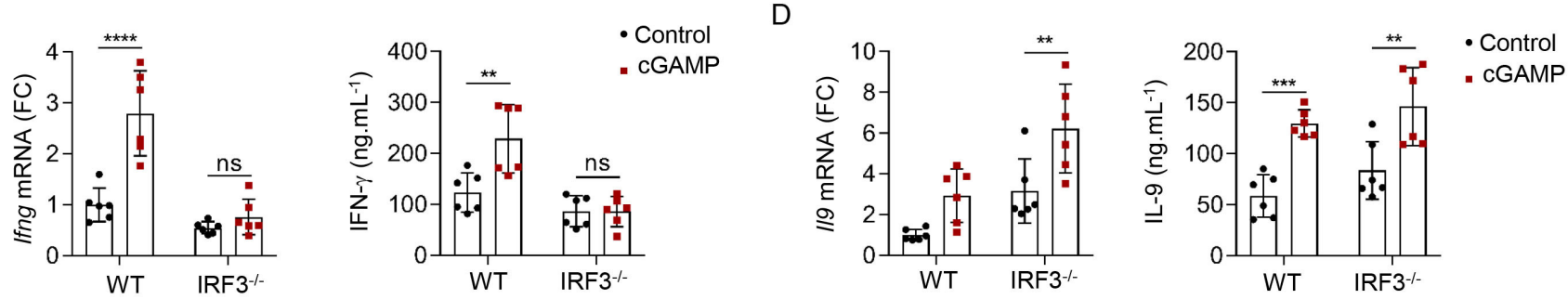

$\mathrm{E}$

$\mathrm{F}$
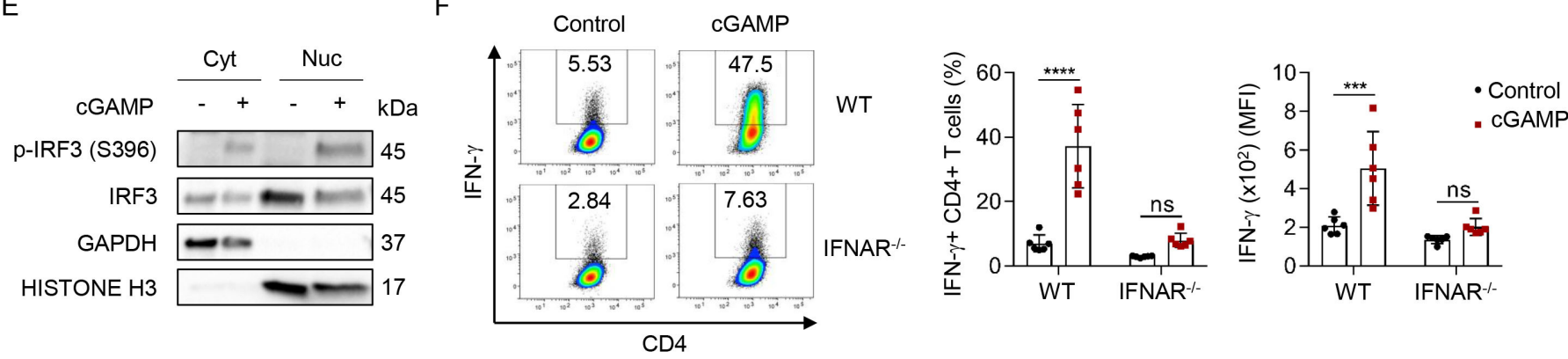

G
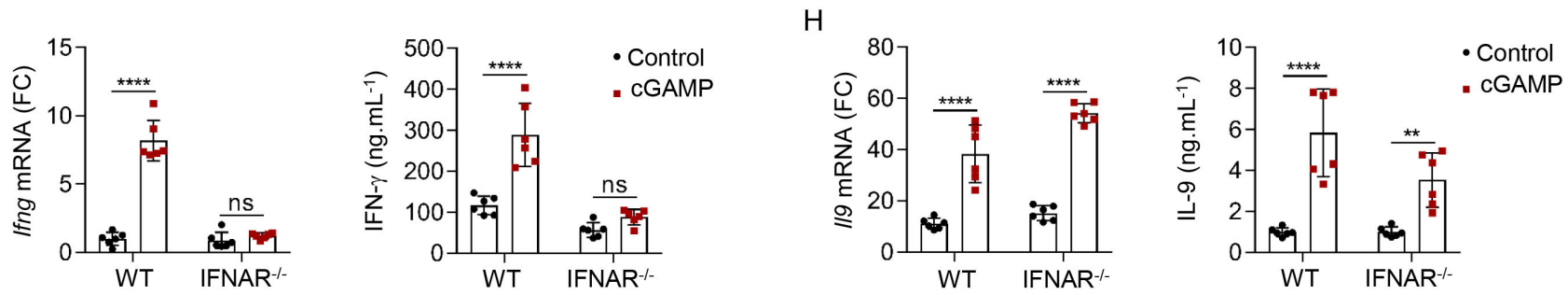

Figure 5 STING signaling enhances $T_{H} 1$ cell differentiation through IRF3 signaling. (A) Ifnb1 mRNA expression (FC, left) and IFN- $\beta$ secretion (right) from WT naive CD4 T cells stimulated with cGAMP or control and polarized into $T_{H} 1$ and $T_{H} 9$ for 16 and 48 hours. Mean \pm SD of replicates from one experiment representative of two independent experiments. (B-D) IFN- $\gamma$ production (representative plots (B, left), frequency (B, middle), and MFI (B right)), Ifng (C) or II9 (D) mRNA expression (FC, left) and IFN- $\gamma$ (C) or IL-9 (D) secretion (right) from WT or IRF3 ${ }^{-/}$naive CD4 T cells stimulated with cGAMP or control and polarized into $\mathrm{T}_{H} 1$ $(B, C)$ or $T_{H} 9$ (D) cells. (E) Cyt and Nuc localization of IRF3 and its phosphorylated form in WT naive CD4 T cells stimulated with cGAMP or control and polarized into $T_{H} 1$ for 16 hours. $(F-H)$ IFN- $\gamma$ production (representative plots ( $F$, left), frequency $(F$, middle), and MFI (F, right)), Ifng $(\mathrm{G})$ or II9 $(\mathrm{H})$ mRNA expression (FC, left) and IFN- $\gamma(\mathrm{G})$ or IL-9 $(\mathrm{H})$ secretion (right) from WT or IFNAR ${ }^{-/-}$ naive CD4 T cells stimulated with cGAMP or control and polarized into $T_{H} 1(F, G)$ or $T_{H} 9(H)$ cells. Mean $\pm S D$ of replicates pooled from two independent experiments $(B-D, F-H)$. $P$ values $\left({ }^{* *} \mathrm{p}<0.01,{ }^{* * *} \mathrm{p}<0.001,{ }^{* * \star *} \mathrm{p}<0.0001\right)$ determined by two-way analysis of variance. cGAMP, 2'3'-cyclic guanosine monophosphate-adenosine monophosphate; Cyt, cytosolic; FC, fold change; IFN- $\gamma$, interferon gamma; IL, interleukin; ns, not significant; MFI, mean fluorescence intensity; Nuc, nuclear; STING, stimulator of interferon genes; WT, wild type. 
activation to affect $\mathrm{T}_{\mathrm{H}} 9$ cell differentiation in the absence of p65. While p65 deficiency reduced IL-9 secretion from differentiating $\mathrm{T}_{\mathrm{H}} 9$ cells, it failed to prevent the ability of cGAMP to enhance $\mathrm{T}_{\mathrm{H}} 9$ cell differentiation (figure $6 \mathrm{~A}$ ). mTOR activation is required for STING-mediated IFN- $\beta$ production in $\mathrm{T}$ cells. ${ }^{48}$ Because activation of mTOR signaling has been reported to be required for $\mathrm{T}_{\mathrm{H}} 9$ cell differentiation, ${ }^{49}$ we tested whether cGAMP could affect mTOR signaling in differentiating $\mathrm{T}_{\mathrm{H}} 9$ cells. We found that cGAMP induced the phosphorylation of the downstream effectors of mTOR pathway P70S6 kinase and $\mathrm{S} 6$ ribosomal protein 48 hours after $\mathrm{T}_{\mathrm{H}} 9$ differentiation initiation and that these phosphorylation events were compromised by the addition of the mTOR inhibitor Rapamycin, suggesting that cGAMP activates mTOR signaling in differentiating $\mathrm{T}_{\mathrm{H}} 9$ cells (figure $6 \mathrm{~B}$ and online supplemental figure S5B). In addition, we found that Rapamycin abrogated the effect of cGAMP on $I l 9$ transcription as well as protein expression and secretion (figure 6C and online supplemental figure S5C). Overall, these results suggest that the ability of cGAMP to enhance the differentiation of $\mathrm{T}_{\mathrm{H}} 9$ cells relies on the activation of mTOR signaling.

$\mathrm{T}_{\mathrm{H}} 9$ cells have recently been proposed to harbor superior anticancer properties compared with $\mathrm{T}_{\mathrm{H}} 1, \mathrm{~T}_{\mathrm{H}} 2$ or $\mathrm{T}_{\mathrm{H}} 17$ in vivo in the $\mathrm{B} 16$ melanoma tumor model. ${ }^{46}$ Given that our results showed that STING ligands enhance $T_{H} 9$ cell differentiation, we examined the ability of naive $\mathrm{CD} 4$ $\mathrm{T}$ cells stimulated with control or cGAMP and differentiated into $\mathrm{T}_{\mathrm{H}} 9$ cells to prevent cancer tumor growth in vivo. For this, B16-OVA tumor cells (ovalbumin-transfected B16-F10 melanoma cells) were first injected subcutaneously into mice. Five days after tumor cell engraftment, mice received an intravenous injection of $\mathrm{T}_{\mathrm{H}} 9$ cells polarized in vitro and derived from OT-II mice, which have a TCR specific for the ovalbumin peptide (323-339) presented by MHC class II molecules. As expected, cGAMP enhanced CD4 T cell-derived $I l 9$ expression from OT-II $\mathrm{T}_{\mathrm{H}} 9$ cells in vitro as compared with control (online supplemental figure S5D). Tumor growth monitoring revealed that cGAMP enhances the antitumor properties of $\mathrm{T}_{\mathrm{H}} 9$ cells upon adoptive transfer (figure 6D). We obtained similar results when B16-OVA cells were injected intravenously, as shown by the reduced number of B16-OVA lung tumor foci in mice which received OT-II $\mathrm{T}_{\mathrm{H}} 9$ cells stimulated with cGAMP compared with control $\mathrm{T}_{\mathrm{H}} 9$ cells (figure $6 \mathrm{E}$ ). Finally, we also tested the anticancer potential of cGAMP-treated $\mathrm{T}_{\mathrm{H}} 9$ cells against B16F-10 cancer cells in vivo using Trp1 transgenic T cells, which recognize tyrosinase-related protein 1 a specific melanoma tumor antigen.$^{50}$ We also found in that setting that cGAMP endowed antigen-specific $\mathrm{T}_{\mathrm{H}} 9$ cells with higher IL-9 production and superior anticancer functions (online supplemental figure S5E,F).

To investigate whether our findings on mouse CD4 T cells are relevant in a human setting, we obtained human blood samples from healthy volunteers, isolated human naive CD4 $\mathrm{T}$ cells and differentiated them into $\mathrm{T}_{\mathrm{H}} 1$ or
$\mathrm{T}_{\mathrm{H}} 9$ cells with IL-12 or TGF- $\beta$ and IL-4, respectively, after stimulation with cGAMP or negative control dinucleotide (control). In line with our results obtained with mouse $\mathrm{T}$ cells, we found that cGAMP promoted human $\mathrm{T}_{\mathrm{H}} 1$ and $\mathrm{T}_{\mathrm{H}} 9$ cell differentiation (figure $6 \mathrm{~F}$ and online supplemental figure $\mathrm{S} 5 \mathrm{G}$ ). Altogether, these results show that the activation of STING signaling with cGAMP not only affects CD4 T-cell biology through the activation of innate immune responses but also cell intrinsically shapes both mouse and human $\mathrm{T}_{\mathrm{H}} 1$ and $\mathrm{T}_{\mathrm{H}} 9$ cell differentiation.

\section{DISCUSSION}

The contribution of CD4 T-cell effector responses to the antitumor immune effects induced by STING ligand administration in the tumor environment was elusive. Here, our results underscore a key role for IFN- $\gamma$ and IL-9 in mediating the anticancer effects following STING ligand administration in vivo. We further show that cellintrinsic STING activation enhances the effector and antitumor functions of $T_{H} 1$ and $T_{H} 9$ cells through two distinct molecular mechanisms, the engagement of the IRF3 and mTOR signaling, respectively. These findings uncover STING as an attractive target to improve CD4 T cell-mediated cancer immunotherapy.

STING was initially characterized to be essential for induction of antiviral immunity, notably because of its ability to promote the secretion of type I IFNs and proinflammatory mediators from DCs and macrophages. ${ }^{16}$ STING-driven activation of the innate immune system promotes adaptive immune responses that not only favor host defense against infections but also can drive the elimination of cancer cells, ${ }^{45}$ indicating the key importance of this molecular pathway for the maintenance of host homeostasis. While the central importance of STING is known to rely on its ability to bridge innate and adaptive immunity on activation, accumulating evidence suggests that adaptive immune cells can directly respond to STING engagement. B cell-intrinsic STING signaling was indeed proposed to promote antibody responses independently of type I IFNs. ${ }^{51}$ Furthermore, STING triggering can drive type I IFN responses in T cells. ${ }^{48}{ }^{52}$ Analysis of knock-in mice STING N153S where STING signaling is constitutively active also revealed that $\mathrm{T}$ cells featured activation of mTOR and an activated phenotype, possibly resulting from a cell-intrinsic effect of the STING mutation. ${ }^{53}$ Whereas initial studies showed that STING activation induces $\mathrm{T}$ cell death, other investigations suggested that the strength of STING signaling differentially affected T-cell activation in a TCR-dependent manner. ${ }^{48}$ STING activation was also recently proposed to enhance the fitness of CD8 T cells, thereby contributing to anticancer immunity. ${ }^{54}$ How STING regulates the differentiation outcome of CD4 T cells, however, was incompletely understood. We find that the nature, potency and dose of STING ligands critically determine the fate of CD4 T cells after STING activation. We also demonstrate that CD4 T-cell polarizing conditions influence the consequences 
A

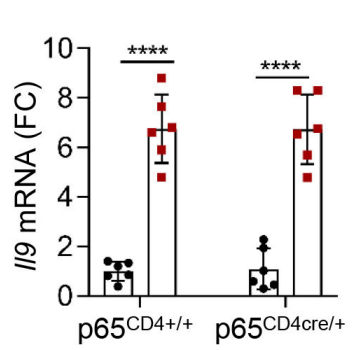

B

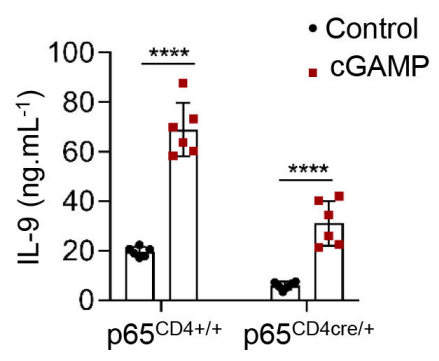

$24 \mathrm{~h}$

$48 \mathrm{~h}$

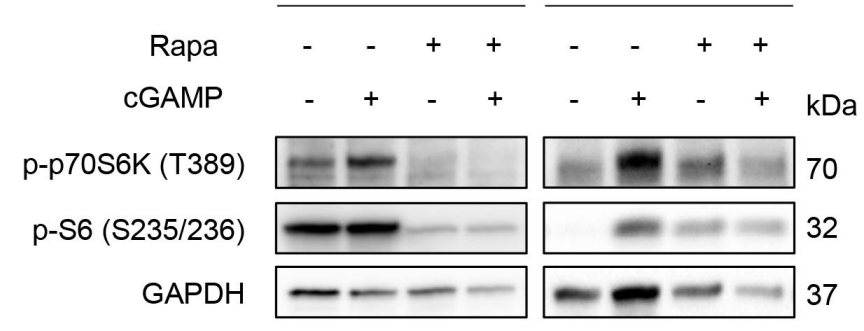

C
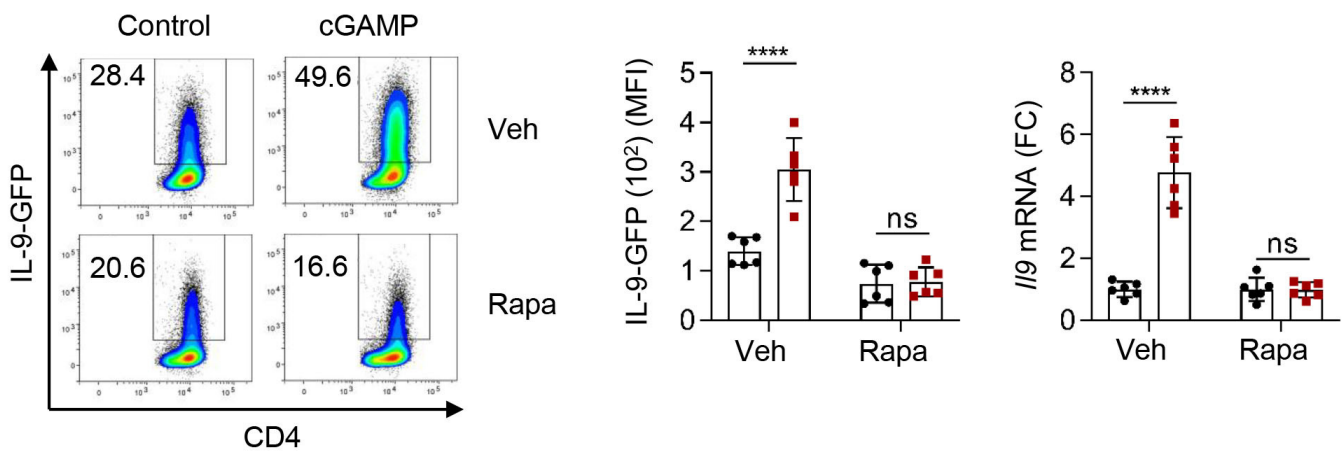

- Control

- cGAMP

D

$\mathrm{E}$
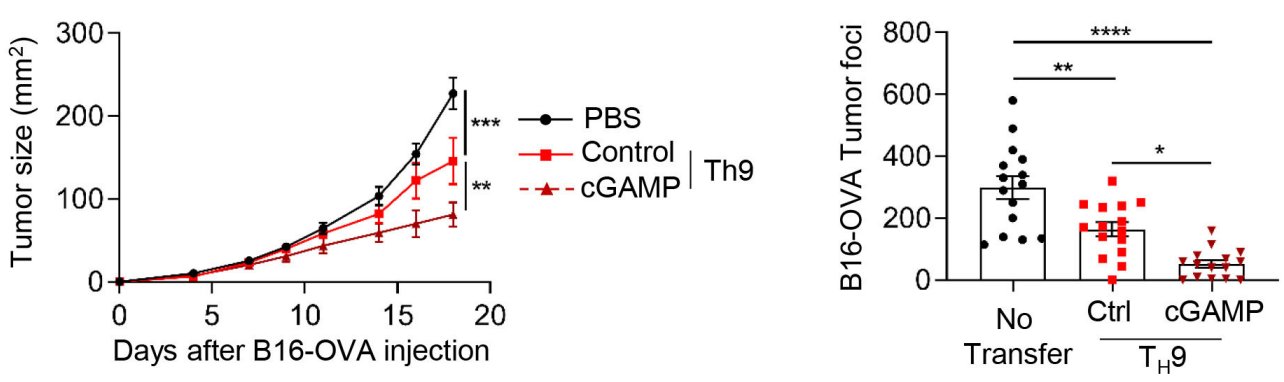

F
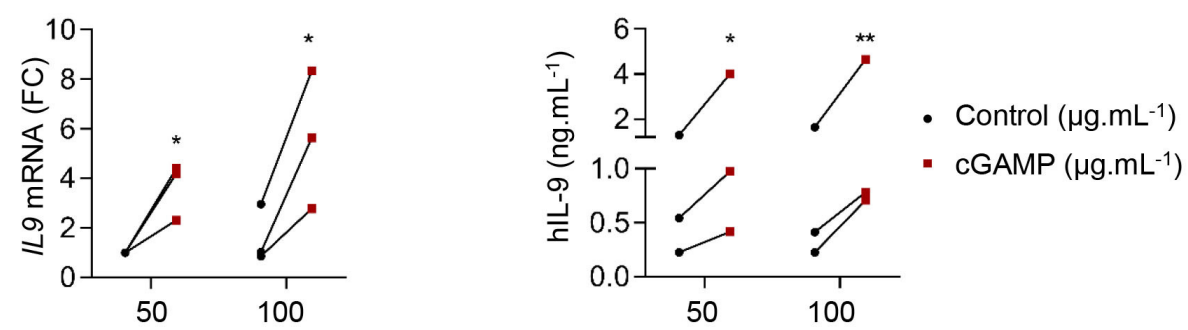

Figure 6 STING signaling enhances $T_{H} 9$ effector functions through activation of mTOR signaling. (A) II9 mRNA expression (FC, left) and IL-9 secretion (right) from $\mathrm{p} 65^{\mathrm{CD} 4+/+}$ or $\mathrm{p} 65^{\mathrm{CD} 4 \mathrm{Cre} /+}$ naive $\mathrm{CD} 4 \mathrm{~T}$ cells stimulated with cGAMP or Ctrl and polarized into $T_{H} 9$ cells. Mean \pm SD of replicates pooled from two independent experiments. (B,C) WT (B) or IL-9-GFP (C) naive CD4 T cells stimulated with cGAMP or Ctrl in the presence of Rapa $(10 \mathrm{nM})$ or Veh and then polarized into $\mathrm{T}_{H} 9$ cells for 24 hours and 48 hours (B) or 3 days (C). (B) Levels of phosphorylated p70S6K (P-p70S6K) and S6 (P-S6). (C) IL-9 production (representative plots, left; MFI, middle; and /I9 mRNA expression (FC), right). Mean \pm SD of replicates pooled from two independent experiments. (D) Tumor size in B16-OVA tumor-bearing mice injected intravenously with PBS (Mock), OT-II T 9 cells or cGAMP-stimulated OTII T 9 cells 5 days after tumor cell inoculation. (E) Tumor foci enumerated 14 days after intravenous injection of B16-OVA tumor cells and intravenous injection of PBS (no transfer), OT-II T 9 cells or cGAMP-stimulated OT-II T 9 cells 1 day after tumor cell inoculation. Mean \pm SEM of $n=11-12$ (D) and $n=15$ (E) mice per group pooled from two independent experiments. (F) IL9 mRNA expression (FC) (left) and hIL-9 secretion (right) from human naive CD4 T cells stimulated with cGAMP or Ctrl and polarized into $T_{H} 9$ cells. Mean of replicates from three independent experiments and each dot represents one donor. $P$ values $\left({ }^{*} p<0.05\right.$, $\left.{ }^{* *} p<0.01,{ }^{* * *} p<0.001,{ }^{* * *} p<0.0001\right)$ determined by two-way ANOVA (A,C,D,F,) or one-way ANOVA (E). ANOVA, analysis of variance; cGAMP, 2'3'-cyclic guanosine monophosphate-AMP; Ctrl, control; FC, fold change; IL, interleukin; ns, not significant; Rapa, rapamycin; STING, stimulator of interferon genes; Veh, vehicle. 
of cell-intrinsic STING activation, as illustrated by a reduced sensitivity to STING-induced cell death of $\mathrm{T}_{\mathrm{H}} 9$ cells compared with $\mathrm{T}_{\mathrm{H}} 1$ cells. This might be due to the control of $\mathrm{T}_{\mathrm{H}} 1$ cell-IFN- $\gamma$ secretion through IRF3/ IFN- $\beta$ /IFNAR axis, which may also affect proliferation and death. ${ }^{24}{ }^{48}$ Although prolonged STING stimulation was proposed to downregulate mTOR signaling in $T_{H} 0$ cells ${ }^{48}$ we instead noted that STING activation stimulated mTOR signaling along with IL-9 production in $\mathrm{T}_{\mathrm{H}} 9$ cells. While this is in line with previous observations that mTOR signaling harnesses $\mathrm{T}_{\mathrm{H}} 9$ cell functions through enhanced glycolytic activity, ${ }^{55}$ this also shows that signaling and outcome of STING activation can be distinct in different T-cell subsets. Our results overall indicate that STING signaling directly shapes the differentiation of $\mathrm{T}_{\mathrm{H}} 1$ and $\mathrm{T}_{\mathrm{H}} 9$ cells, thereby lending further support to a determinant function of cell-intrinsic STING signaling in T-cell fate. Whether STING activation additionally affects T-cell persistence in vivo warrants further investigations.

$\mathrm{T}_{\mathrm{H}} 9$ cells have emerged as a unique CD4 T-cell subset of particular interest for adoptive cell therapy of cancer. Compared with other CD4 T-cell subsets, murine $\mathrm{T}_{\mathrm{H}} 9$ cells elicit the highest antitumor response upon adoptive transfer because of their superior persistence, cytolytic functions and resistance to T-cell dysfunction. ${ }^{4}$ Importantly, human chimeric antigenic receptor (CAR) T cells cultured under $\mathrm{T}_{\mathrm{H}}$ 9-polarizing conditions exert enhanced antitumor activity compared with cells cultured with IL-2, underscoring the clinical relevance of $\mathrm{T}_{\mathrm{H}} 9$ cells for cancer therapy. ${ }^{56}$ Previous studies reported that cell-extrinsic signals could favor $\mathrm{T}_{\mathrm{H}} 9$ cell differentiation. Indeed, proinflammatory factors secreted by APCs as well as engagement of costimulatory molecules like OX40 were shown to favor $\mathrm{T}_{\mathrm{H}} 9$ cell differentiation. ${ }^{1257}$ Here, we extend these findings by identifying how $\mathrm{T}_{\mathrm{H}} 9$ cell differentiation can be regulated cell intrinsically following the triggering of the STING signaling pathway, which favors the promotion of inflammation. Our results show not only that CD4 T cellintrinsic STING activation potentiates $\mathrm{T}_{\mathrm{H}} 9$ cell antitumor effect upon adoptive transfer in mice but also that human $\mathrm{T}_{\mathrm{H}} 9$ cell differentiation can be enhanced through STING activation. While STING agonists were recently suggested to synergize with CAR T cells and enhance their ability to control tumor growth, ${ }^{58}$ our findings provide impetus for studying the relevance of STING activation in $\mathrm{T}_{\mathrm{H}} 9$ cells in the context of adoptive T-cell therapy for cancer.

The ability of STING to regulate adaptive immunity is actually reminiscent of the cell-intrinsic activity of toll-like receptors (TLRs) in CD4 T cells. Indeed, while TLR activation was initially solely considered to bridge innate and adaptive immunity, it is now clear that TLR can also directly affect CD4 T-cell responses. ${ }^{59}{ }^{60}$ Importantly, synthetic CDNs, nanoparticulate STING agonists, as well as small-molecule STING inhibitors have recently been shown to exhibit functional activities in vivo. ${ }^{61-63}$ Our findings could thus be therapeutically exploited to manipulate CD4 T-cell responses in infections, inflammatory diseases, and cancers.

\section{Author affiliations}

${ }^{1}$ INSERM, U1231, Dijon, France

${ }^{2}$ UFR Sciences de Santé, Université Bourgogne Franche-Comté, Dijon, France ${ }^{3}$ INSERM, UMR-S 1193, Université Paris-Saclay, Châtenay-Malabry, France

${ }^{4}$ Department of Molecular and Integrative Physiology, University of Illinois at Urbana Champaign, Urbana, IL, USA

${ }^{5}$ INSERM UMR - S1109, Department of Clinical Immunology and Internal Medicine, National Reference Center for Systemic Autoimmune Diseases (CNR RES0), Tertiary Center for Primary Immunodeficiency, Hôpitaux Universitaires de Strasbourg, Université de Strasbourg, Strasbourg, France

${ }^{6}$ Laboratoire d'ImmunoRhumatologie Moléculaire, GENOMAX platform, INSERM

UMR_S 1109, Faculté de Médecine, Fédération Hospitalo-Universitaire

OMICARE, Fédération de Médecine Translationnelle de Strasbourg (FMTS), LabEx TRANSPLANTEX, Strasbourg, France

${ }^{7}$ Department of Biology and Pathology of Tumors, Centre Georges François Leclerc, Dijon, France

${ }^{8}$ Department of Immunobiology, Yale University School of Medicine, New Heaven, CT, USA

${ }^{9}$ UMR 7355, Experimental and Molecular Immunology and Neurogenetics, CNRS, Orléans, France

${ }^{10}$ Department of Immunology, Institute of Infectious Disease and Molecular Medicine, University of Cape Town, Cape Town, South Africa

${ }^{11}$ Anticancer Discovery from Pets to People Theme, University of Illinois Urbana-

Champaign, Cancer Center at Illinois, Urbana Champaign, Illinois, USA

${ }^{12}$ University of Illinois Cancer Center, University of Illinois at Chicago, Chicago, IL, USA

${ }^{13}$ INSERM UMR-S1109, Department of Clinical Immunology and Internal Medicine, National Reference Center for Systemic Autoimmune Diseases (CNR RESO),

Tertiary Center for Primary Immunodeficiency, Faculty of Pharmacy, Université de Strasbourg, Strasbourg, France

${ }^{14}$ Centre d'Immunologie de Marseille-Luminy, Université Aix-Marseille, INSERM, CNRS, Marseille, France

${ }^{15}$ INSERM, U1100, Tours, France

${ }^{16}$ Faculté de Médecine, Université de Tours, Tours, France

\section{Twitter Romain Boidot @boidot_romain}

Acknowledgements This work was possible with help from Flow Cytometry Core Facility/INSERM UMR1231, at the University of Burgundy and the Biomax Facility/ Centre de Recherche d'Immunologie et d'Hématologie Strasbourg.

Contributors IB-L, EJ, TRV, AR, LDZ, TM, AM, SHS, and CP performed in vitro and/or in vivo experiments; SG, RC, CR and RB performed and analyzed NGS experiments; ABG, DV, FV, RAF, BR, ERN, PS-S, and TL provided materials and suggestions for the study. IB-L, EJ, TRV, and LA wrote the paper and supervised the study. LA acts as the guarantor of the study.

Funding The authors were supported by grants from the Fondation de France (LA, TRV, and ABG), the Fondation pour la Recherche Médicale (ARF20170938687) (EJ), the Conseil Régional de Bourgogne and FEDER (LA), the Agence Nationale de la Recherche (ANR-11-LABX-0021) (TM, AR, AM, ABG, and LA), the LabEx MAblmprove (ANR-10-LABX-53-01) (LA), as well as (ANR-14-CE14-0026-04, Lumugene; and ANR 19-CE15-0028-01, Lympho-sting) (PS-S), The Fondation ARC (D0C20190509200) (IB-L), the National Cancer Institute of the National Institutes of Health (R01CA234025) (ERN and SHS), the Department of Defense Breast Cancer Research Program (BC171214) (ERN and SHS), and the Embassy of France in the USA, 2018-2019 STEM Chateaubriand Fellowship (SHS). This project has received funding from the European Research Council under the European Union's Horizon 2020 research and innovation program (grant agreement number 677251).

Competing interests LA performed consultancy work for Roche, Merck, BristolMyers Squibb, and Orega Biotech and was a recipient of a research grant from Sanofi.

\section{Patient consent for publication Not applicable.}

Ethics approval All animals were bred and maintained according to both the FELASA and the Animal Experimental Ethics Committee Guidelines (University of Burgundy and TAAM, Orléans, France). The Ethics Committee for Animal Welfare of the University of Burgundy and the French Ministry of Higher Education and Research approved all animal experiments (reference 10626).

Provenance and peer review Not commissioned; externally peer reviewed. 
Data availability statement Data are available upon reasonable request. RNAseq data are deposited in the Gene Expression Omnibus database under accession number GSE147300.

Supplemental material This content has been supplied by the author(s). It has not been vetted by BMJ Publishing Group Limited (BMJ) and may not have been peer-reviewed. Any opinions or recommendations discussed are solely those of the author(s) and are not endorsed by BMJ. BMJ disclaims all liability and responsibility arising from any reliance placed on the content. Where the content includes any translated material, BMJ does not warrant the accuracy and reliability of the translations (including but not limited to local regulations, clinical guidelines, terminology, drug names and drug dosages), and is not responsible for any error and/or omissions arising from translation and adaptation or otherwise.

Open access This is an open access article distributed in accordance with the Creative Commons Attribution 4.0 Unported (CC BY 4.0) license, which permits others to copy, redistribute, remix, transform and build upon this work for any purpose, provided the original work is properly cited, a link to the licence is given, and indication of whether changes were made. See https://creativecommons.org/ licenses/by/4.0/.

\section{ORCID iDs}

Isis Benoit-Lizon http://orcid.org/0000-0003-1187-6828

Elise Jacquin http://orcid.org/0000-0003-0368-4113

Alvaro Baeza Garcia http://orcid.org/0000-0001-6032-7847

Lionel Apetoh http://orcid.org/0000-0002-2774-438X

\section{REFERENCES}

1 Alspach E, Lussier DM, Miceli AP, et al. MHC-Il neoantigens shape tumour immunity and response to immunotherapy. Nature 2019;574:696-701.

2 Hunder NN, Wallen $\mathrm{H}$, Cao J, et al. Treatment of metastatic melanoma with autologous CD4+ T cells against NY-ESO-1. N Engl $J$ Med 2008;358:2698-703.

3 Hsieh CS, Macatonia SE, Tripp CS, et al. Development of TH1 CD4+ $\mathrm{T}$ cells through IL-12 produced by Listeria-induced macrophages. Science 1993;260:547-9.

4 Lu Y, Wang Q, Xue G, et al. TH9 cells represent a unique subset of $\mathrm{CD}^{+} \mathrm{T}$ cells endowed with the ability to eradicate advanced tumors. Cancer Cell 2018;33-1048-60.

5 Végran $\mathrm{F}$, Berger $\mathrm{H}$, Boidot R, et al. The transcription factor IRF1 dictates the IL-21-dependent anticancer functions of TH9 cells. Nat Immunol 2014:15:758-66.

6 Purwar R, Schlapbach C, Xiao S, et al. Robust tumor immunity to melanoma mediated by interleukin-9-producing T cells. Nat Med 2012;18:1248-53.

7 Dardalhon V, Awasthi A, Kwon H, et al. IL-4 inhibits TGF-betainduced Foxp3+ T cells and, together with TGF-beta, generates IL-9+ IL-10+ Foxp3(-) effector T cells. Nat Immunol 2008;9:1347-55.

8 Jiang $Y$, Chen J, Bi E, et al. TNF- $\alpha$ enhances Th9 cell differentiation and antitumor immunity via TNFR2-dependent pathways. $J$ Immunother Cancer 2019;7:28.

9 Xue G, Jin G, Fang J, et al. IL-4 together with IL-1 $\beta$ induces antitumor Th9 cell differentiation in the absence of TGF- $\beta$ signaling. Nat Commun 2019;10:1376.

10 Benoit-Lizon I, Apetoh L. Harnessing Th9 cells in cancer immunotherapy. Semin Immunol 2021;52:101477.

$11 \mathrm{Kim}$ I-K, Kim B-S, Koh C-H, et al. Glucocorticoid-induced tumor necrosis factor receptor-related protein co-stimulation facilitates tumor regression by inducing IL-9-producing helper T cells. Nat Med 2015;21:1010-7.

12 Zhao Y, Chu X, Chen J, et al. Dectin-1-activated dendritic cells trigger potent antitumour immunity through the induction of Th9 cells. Nat Commun 2016;7:12368.

13 Banchereau J, Steinman RM. Dendritic cells and the control of immunity. Nature 1998;392:245-52.

14 Barbalat R, Ewald SE, Mouchess ML, et al. Nucleic acid recognition by the innate immune system. Annu Rev Immunol 2011;29:185-214.

15 Ishikawa H, Barber GN. STING is an endoplasmic reticulum adaptor that facilitates innate immune signalling. Nature 2008;455:674-8.

16 Ishikawa H, Ma Z, Barber GN. STING regulates intracellular DNAmediated, type I interferon-dependent innate immunity. Nature 2009;461:788-92.

17 Demaria O, De Gassart A, Coso S, et al. STING activation of tumor endothelial cells initiates spontaneous and therapeutic antitumor immunity. Proc Natl Acad Sci U S A 2015;112:15408-13.
18 Deng L, Liang $\mathrm{H}$, Xu M, et al. STING-dependent cytosolic DNA sensing promotes radiation-induced type I interferondependent antitumor immunity in immunogenic tumors. Immunity 2014;41:843-52

19 Sivick KE, Desbien AL, Glickman LH, et al. Magnitude of therapeutic STING activation determines $\mathrm{CD}^{+} \mathrm{T}$ cell-mediated anti-tumor immunity. Cell Rep 2018;25:e3075:3074-85.

20 Ramanjulu JM, Pesiridis GS, Yang J, et al. Design of amidobenzimidazole STING receptor agonists with systemic activity. Nature 2018;564:439-43.

21 Kwon J, Bakhoum SF. The cytosolic DNA-sensing cGAS-STING pathway in cancer. Cancer Discov 2020;10:26-39.

22 Monroe KM, Yang Z, Johnson JR, et al. IFI16 DNA sensor is required for death of lymphoid CD4 T cells abortively infected with HIV. Science 2014;343:428-32.

23 Berg RK, Rahbek SH, Kofod-Olsen E, et al. T cells detect intracellular DNA but fail to induce type I IFN responses: implications for restriction of HIV replication. PLoS One 2014;9:e84513.

24 Gulen MF, Koch U, Haag SM, et al. Signalling strength determines proapoptotic functions of STING. Nat Commun 2017;8:427.

25 Cerboni S, Jeremiah N, Gentili M, et al. Intrinsic antiproliferative activity of the innate sensor STING in T lymphocytes. J Exp Med 2017;214:1769-85

26 Larkin B, llyukha V, Sorokin $\mathrm{M}$, et al. Cutting edge: activation of STING in T cells induces type I IFN responses and cell death. J Immunol 2017;199:397-402.

27 Wu J, Dobbs N, Yang K, et al. Interferon-independent activities of mammalian sting mediate antiviral response and tumor immune evasion. Immunity 2020;53:115-26.

28 Licona-Limón P, Henao-Mejia J, Temann AU, et al. Th9 cells drive host immunity against gastrointestinal worm infection. Immunity 2013;39:744-57.

29 Bouis D, Kirstetter P, Arbogast F, et al. Severe combined immunodeficiency in stimulator of interferon genes (STING) V154M/ wild-type mice. J Allergy Clin Immunol 2019;143:e715:712-25.

30 Kim D, Pertea G, Trapnell C, et al. TopHat2: accurate alignment of transcriptomes in the presence of insertions, deletions and gene fusions. Genome Biol 2013;14:R36.

31 Trapnell C, Williams BA, Pertea G, et al. Transcript assembly and quantification by RNA-seq reveals unannotated transcripts and isoform switching during cell differentiation. Nat Biotechnol 2010;28:511-5.

32 Bolger AM, Lohse M, Usadel B. Trimmomatic: a flexible trimmer for illumina sequence data. Bioinformatics 2014;30:2114-20.

33 Love MI, Huber W, Anders S. Moderated estimation of fold change and dispersion for RNA-seq data with DESeq2. Genome Biol 2014;15:550.

34 Mootha VK, Lindgren CM, Eriksson K-F, et al. PGC-1alpharesponsive genes involved in oxidative phosphorylation are coordinately downregulated in human diabetes. Nat Genet 2003;34:267-73.

35 Subramanian A, Tamayo P, Mootha VK, et al. Gene set enrichment analysis: a knowledge-based approach for interpreting genome-wide expression profiles. Proc Natl Acad Sci U S A 2005;102:15545-50.

36 Chen EY, Tan CM, Kou Y, et al. Enrichr: interactive and collaborative HTML5 gene list enrichment analysis tool. BMC Bioinformatics 2013;14:128.

37 Kuleshov MV, Jones MR, Rouillard AD, et al. Enrichr: a comprehensive gene set enrichment analysis web server 2016 update. Nucleic Acids Res 2016;44:W90-7.

38 Ahrends T, Spanjaard A, Pilzecker B, et al. CD4 ${ }^{+}$T cell help confers a cytotoxic $T$ cell effector program including coinhibitory receptor downregulation and increased tissue invasiveness. Immunity 2017;47:e845:848-61.

39 Foote JB, Kok M, Leatherman JM, et al. A STING agonist given with OX40 receptor and PD-L1 modulators primes immunity and reduces tumor growth in tolerized mice. Cancer Immunol Res 2017;5:468-79.

40 Qin Z, Schwartzkopff J, Pradera F, et al. A critical requirement of interferon gamma-mediated angiostasis for tumor rejection by CD8+ T cells. Cancer Res 2003;63:4095-100.

41 Chen Q, Sun L, Chen ZJ. Regulation and function of the cGAS-STING pathway of cytosolic DNA sensing. Nat Immunol 2016;17:1142-9.

42 Wang Y, Lian Q, Yang B, et al. TRIM30 $\alpha$ is a negative-feedback regulator of the intracellular DNA and DNA virus-triggered response by targeting STING. PLoS Pathog 2015;11:e1005012.

43 Zhang $M$, Zhang M-X, Zhang Q, et al. USP18 recruits USP20 to promote innate antiviral response through deubiquitinating STING/ MITA. Cell Res 2016;26:1302-19. 
44 Roberts ZJ, Goutagny N, Perera P-Y, et al. The chemotherapeutic agent DMXAA potently and specifically activates the TBK1-IRF-3 signaling axis. J Exp Med 2007;204:1559-69.

45 Barber GN. STING: infection, inflammation and cancer. Nat Rev Immunol 2015;15:760-70.

46 Abe T, Barber GN. Cytosolic-DNA-mediated, STING-dependent proinflammatory gene induction necessitates canonical NF-kappaB activation through TBK1. J Virol 2014;88:5328-41.

47 Jash A, Sahoo A, Kim G-C, et al. Nuclear factor of activated T cells 1 (NFAT1)-induced permissive chromatin modification facilitates nuclear factor-kappaB (NF-kappaB)-mediated interleukin-9 (IL-9) transactivation. J Biol Chem 2012;287:15445-57.

48 Imanishi T, Unno M, Kobayashi W, et al. Reciprocal regulation of STING and TCR signaling by mTORC1 for T-cell activation and function. Life Sci Alliance 2019;2:e201800282 doi:10.26508/ Isa.201800282

49 Wang Y, Bi Y, Chen X, et al. Histone Deacetylase SIRT1 Negatively Regulates the Differentiation of Interleukin-9-Producing CD4(+) T Cells. Immunity 2016;44:1337-49.

50 Muranski P, Borman ZA, Kerkar SP, et al. Th17 cells are long lived and retain a stem cell-like molecular signature. Immunity 2011;35:972-85.

51 Walker MM, Crute BW, Cambier JC, et al. B cell-intrinsic STING signaling triggers cell activation, synergizes with B cell receptor signals, and promotes antibody responses. J Immunol 2018;201:2641-53.

52 Imanishi T, Saito T. T cell co-stimulation and functional modulation by innate signals. Trends Immunol 2020;41:200-12.
53 Warner JD, Irizarry-Caro RA, Bennion BG, et al. STING-associated vasculopathy develops independently of IRF3 in mice. J Exp Med 2017;214:3279-92

54 Li W, Lu L, Lu J. cGAS-STING-mediated DNA sensing maintains CD8 + T cell stemness and promotes antitumor T cell therapy. Sci Trans/ Med 2020;12.

55 Roy S, Awasthi A. ATP triggers human Th9 cell differentiation via nitric oxide-mediated mTOR-HIF1 $\alpha$ pathway. Front Immunol 2019;10:1120.

56 Liu L, Bi E, Ma X, et al. Enhanced CAR-T activity against established tumors by polarizing human T cells to secrete interleukin-9. Nat Commun 2020;11:5902.

57 Xiao X, Balasubramanian S, Liu W, et al. OX40 signaling favors the induction of $\mathrm{T}(\mathrm{H}) 9$ cells and airway inflammation. Nat Immunol 2012;13:981-90.

$58 \mathrm{Xu} \mathrm{N}$, Palmer DC, Robeson AC, et al. STING agonist promotes CAR T cell trafficking and persistence in breast cancer. J Exp Med 2021;218.

59 Reynolds JM, Dong C. Toll-like receptor regulation of effector T lymphocyte function. Trends Immunol 2013;34:511-9.

60 Caramalho I, Lopes-Carvalho T, Ostler D, et al. Regulatory T cells selectively express toll-like receptors and are activated by lipopolysaccharide. J Exp Med 2003;197:403-11.

61 Corrales L, Glickman LH, McWhirter SM, et al. Direct activation of sting in the tumor microenvironment leads to potent and systemic tumor regression and immunity. Cell Rep 2015;11:1018-30.

62 Haag SM, Gulen MF, Reymond L, et al. Targeting STING with covalent small-molecule inhibitors. Nature 2018;559:269-73.

63 Luo M, Wang H, Wang Z, et al. A STING-activating nanovaccine for cancer immunotherapy. Nat Nanotechnol 2017;12:648-54. 